\title{
Severe fever with thrombocytopenia syndrome virus targets B cells in lethal human infections
}

\author{
Tadaki Suzuki, ${ }^{1}$ Yuko Sato, ${ }^{1}$ Kaori Sano, ${ }^{1,2}$ Takeshi Arashiro, ${ }^{1}$ Harutaka Katano, ${ }^{1}$ Noriko Nakajima, ${ }^{1}$ Masayuki Shimojima, ${ }^{3}$ \\ Michiyo Kataoka, ${ }^{1}$ Kenta Takahashi, ${ }^{1}$ Yuji Wada, ${ }^{1}$ Shigeru Morikawa, ${ }^{4}$ Shuetsu Fukushi, ${ }^{3}$ Tomoki Yoshikawa, ${ }^{3}$ \\ Masayuki Saijo, ${ }^{3}$ and Hideki Hasegawa ${ }^{1,2,5}$ \\ 'Department of Pathology, National Institute of Infectious Diseases, Shinjuku, Tokyo, Japan. ²Division of Infectious Diseases Pathology, Department of Global Infectious Diseases, Tohoku Graduate School \\ of Medicine, Sendai, Miyagi, Japan. ${ }^{3}$ Department of Virology I, National Institute of Infectious Diseases, Musashimurayama, Tokyo, Japan. ${ }^{4}$ Department of Veterinary Science, National Institute of Infectious \\ Diseases, Shinjuku, Tokyo, Japan. ${ }^{5}$ Clobal Virus Network, Baltimore, Maryland, USA.
}

\begin{abstract}
Severe fever with thrombocytopenia syndrome (SFTS) is an emerging hemorrhagic fever caused by a tick-borne banyangvirus and is associated with high fatality. Despite increasing incidence of SFTS and serious public health concerns in East Asia, the pathogenesis of lethal SFTS virus (SFTSV) infection in humans is not fully understood. Numbers of postmortem examinations to determine target cells of the viral infection have so far been limited. Here we showed that B cells differentiating into plasmablasts and macrophages in secondary lymphoid organs were targets for SFTSV at the end stage of lethal infection, and the majority of SFTSV-infected cells were B cell-lineage lymphocytes. In affected individuals, B cell-lineage lymphocytes with SFTSV infection were widely distributed in both lymphoid and nonlymphoid organs, and infiltration of these cells into the capillaries of the organs could be observed occasionally. Moreover, a human plasmablastic lymphoma cell line, PBL-1, was susceptible to SFTSV propagation and had a similar immunophenotype to that of target cells of SFTSV in fatal SFTS. PBL-1 can therefore provide a potential in vitro model for human SFTSV infection. These results extend our understanding of the pathogenesis of human lethal SFTSV infection and can facilitate the development of SFTSV countermeasures.
\end{abstract}

\section{Introduction}

Severe fever with thrombocytopenia syndrome (SFTS) is an emerging viral hemorrhagic fever caused by SFTS virus (SFTSV), which belongs to the genus Banyangvirus in the family Phenuiviridae and the order Bunyavirales (1). SFTSV is genetically closely related to Heartland virus (HRTV), which has been found to cause a severe, and occasionally fatal, febrile illness in humans in cases in the US (2). Increasing incidence of SFTS has led to serious public health concerns in countries throughout East Asia, including China, Japan, and South Korea (3-9). The typical clinical course of SFTS has 3 distinct periods that are characterized according to disease progression: a fever stage, a multiple-organ-dysfunction (MOD) stage, and a convalescence stage (4, 10-14). Clinical manifestations of the fever stage include a high fever, headache, fatigue, myalgia, and gastrointestinal symptoms with marked thrombocytopenia, leukocytopenia, lymphadenopathy, and high serum viral load. The fever stage is followed by progressive worsening of MOD, leading to fatality, or by self-limiting MOD and survival. MOD develops in most cases approximately 5 days after the

Related Commentary: p. 598

Conflict of interest: The authors have declared that no conflict of interest exists. Copyright: ( 2020, American Society for Clinical Investigation.

Submitted: March 28, 2019; Accepted: October 22, 2019; Published: January 6, 2020

Reference information: / Clin Invest. 2020;130(2):799-812.

https://doi.org/10.1172/JCl129171. onset of illness. The serum viral load gradually falls in individuals with self-limiting illness, but remains high in fatal illness. Clinical symptoms of the MOD phase include hemorrhagic manifestation, neurological symptoms, disseminated intravascular coagulation, and sustained thrombocytopenia. In mild and self-limiting disease, SFTS resolves in the subsequent convalescence stage. Despite the high awareness within the medical community in SFTS-endemic areas, and the use of antiviral therapy such as ribavirin, the case fatality rate of SFTS is still as high as $15 \%$, which is the same as other severe viral diseases including viral hemorrhagic fevers (15). In SFTS, inflammatory cytokine storms $(11,16-19)$ as well as impairment of immune responses including innate immunity (14, 20-25), antiviral T cell function (26), and antiviral humoral responses (27) have important roles in the pathogenic progress of lethal infections. Immune impairment and high viral loads are also characteristics of several other viral hemorrhagic fevers (28), but these diseases differ in terms of pathology and pathogenesis, about which little is known for SFTS. Models of immunodeficient mice $(14,22,24,29)$ or mice treated with an immunosuppressive agent (30) have provided evidence that the innate immune response is essential for development of fatal SFTS. In these models, immune cells such as macrophages, immature B cells, and fibroblastic reticular cells in secondary lymphoid organs (SLOs) have been identified as targets of SFTSV infection (14). Results in a ferret model have also demonstrated that delayed innate immune responses and progressive viral replication are involved in SFTSV-induced mortality (31). These results in animal models 
A

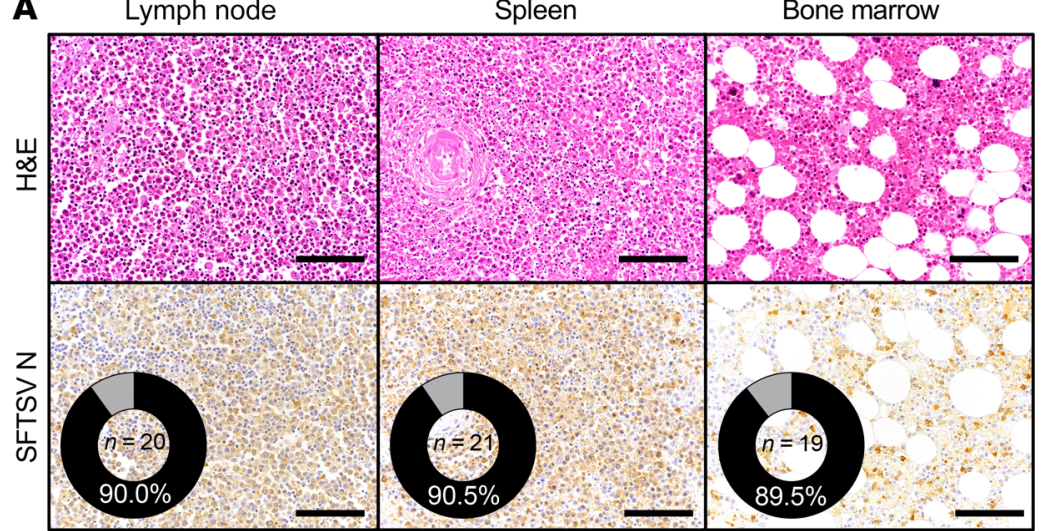

B

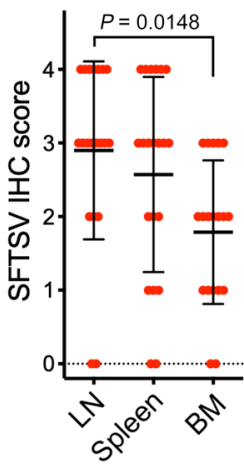

C

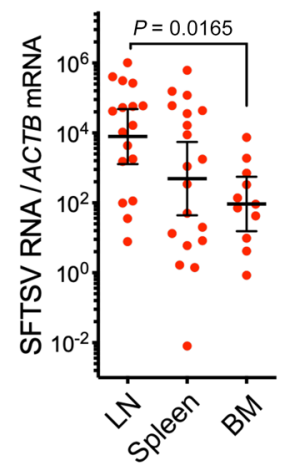

Figure 1. Secondary lymphoid organs are the primary viral targets in human fatal SFTS. (A) Representative pathological images of lymph nodes, spleen, and bone marrow of individuals who died from SFTS, with H\&E staining (upper panels) or immunohistochemistry (IHC) for SFTSV N protein (lower panels). Pie charts show the number of individuals whose tissues were analyzed and the percentage in whose tissues SFTSV antigen was detected by IHC. Scale bars: $100 \mu \mathrm{m}$. (B) SFTSV IHC scores for tissue sections of lymphoid organs. When multiple FFPE blocks of an organ from 1 individual were available, only the section with the highest number of SFTSV+ cells was analyzed. The IHC score for lymph nodes (LNs) was significantly higher than for bone marrow (BM). Scatter plots show the mean \pm SD $(n=20,21$, and 19 for LNs, spleen, and BM, respectively). The $P$ values were calculated with 1-way ANOVA followed by Holm-Sidak's multiple-comparisons test. (C) SFTSV viral RNA loads in the tissues of lymphoid organs. Scatter plots show the relative levels of RNA in tissue sections, quantified by real-time quantitative RT-PCR for SFTSV S segment and human ACTB mRNA (for normalization of RNA extraction). Ceometric means $\pm 95 \%$ confidence intervals are also shown $(n=17,19$, and 11 for $L N s$, spleen, and BM, respectively). The $P$ values were calculated with nonparametric 1-way ANOVA (Kruskal-Wallis test) followed by Dunn's multiple-comparison test. Relative SFTSV RNA copy numbers in LNs were significantly higher than in BM.

are consistent with clinical observations in severe or fatal human SFTS, and demonstrate the importance of host immune systems in determining the severity of SFTS. However, the nature of the disturbance of host immune responses in severe or fatal human SFTS has not previously been identified.

Pathological studies have been important for the discovery and advancement of our knowledge of viral hemorrhagic fevers (32). Autopsies following fatal human infections have provided valuable insights into the pathogenic mechanisms underpinning disease severity. In addition, elucidation of the cell and tissue tropism associated with mortality can explain viral lethality (33). Results from histopathological studies have shown that necrotizing lymphadenitis and prominent hemophagocytosis are the pathological characteristics of fatal SFTS, and large atypical immunoblastic cells are major infected cells in the lymph nodes, spleen, and bone marrow $(6,18,34-37)$. However, detailed characterization of the viral target cells and tissues is required to understand the pathogenic mechanisms of lethal SFTSV infection.

In the present study, we first evaluated organs obtained from 22 autopsies to determine the viral cell and tissue tropism in lethal human SFTSV infection. Next, we demonstrated that the majority of SFTSV-infected cells in lymphoid and nonlymphoid organs in fatal SFTS are class-switched B cells with immunophenotypic resemblance to plasmablasts. Finally, we examined various human B cell lines for susceptibility to SFTSV, to determine the target cells of plasmablast-lineage differentiation.

\section{Results}

SLOs are more frequently infected with SFTSV than bone marrow. Previous pathological examinations of fatal SFTS revealed that major target cells of viral infection are large hematopoietic cells in lymphoid organs such as the lymph nodes, spleen, and bone marrow $(6,18,34)$. However, reported numbers of infected cells in these organs vary considerably. To accurately determine the specificity of SFTSV infection for particular target organs, the presence of SFTSV-infected cells within lymphoid organs obtained postmortem from 22 individuals who died as a result of SFTS was analyzed by immunohistochemistry (IHC) with an antibody against SFTSV nucleocapsid protein (N). The large hematopoietic cells that were positive for SFTSV $\mathrm{N}$ were detected within the lymph nodes, spleen, or bone marrow in approximately $90 \%$ of the individuals (Figure 1A). The numbers of cells that were positive for viral antigen in each lymphoid organ were estimated by IHC scoring (Supplemental Figure 1; supplemental material available online with this article; https://doi.org/10.1172/JCI129171DS1). IHC scores of the lymph nodes were significantly higher than those of the bone marrow (Figure 1B), which was corroborated by comparison of SFTSV RNA loads (Figure 1C). These observations suggested that the SLOs, such as the lymph nodes and the spleen, which are the specialized sites for antigen recognition and initiation of immune responses coordinated by mature lymphocytes, contained the largest numbers of SFTSV-infected cells among the lymphoid organs, so that mature lymphocytes might be the most susceptible to SFTSV infection in fatal SFTS.

The immunophenotype of SFTSV-infected cells in lymph nodes is compatible with activated mature B cells differentiated to the plasmablast lineage. To determine the cell types infected with SFTSV in the lymph nodes in fatal SFTS, IHC for cellular and SFTSV markers was performed. Numerous SFTSV-antigen ${ }^{+}$cells were detected, and cells in the same area of the lymph node stained with cellular markers for T cells (CD3), B cells (CD79a and CD20), and macrophages (CD163) (Figure 2A). Notably, MUM1, BLIMP1, and 
A
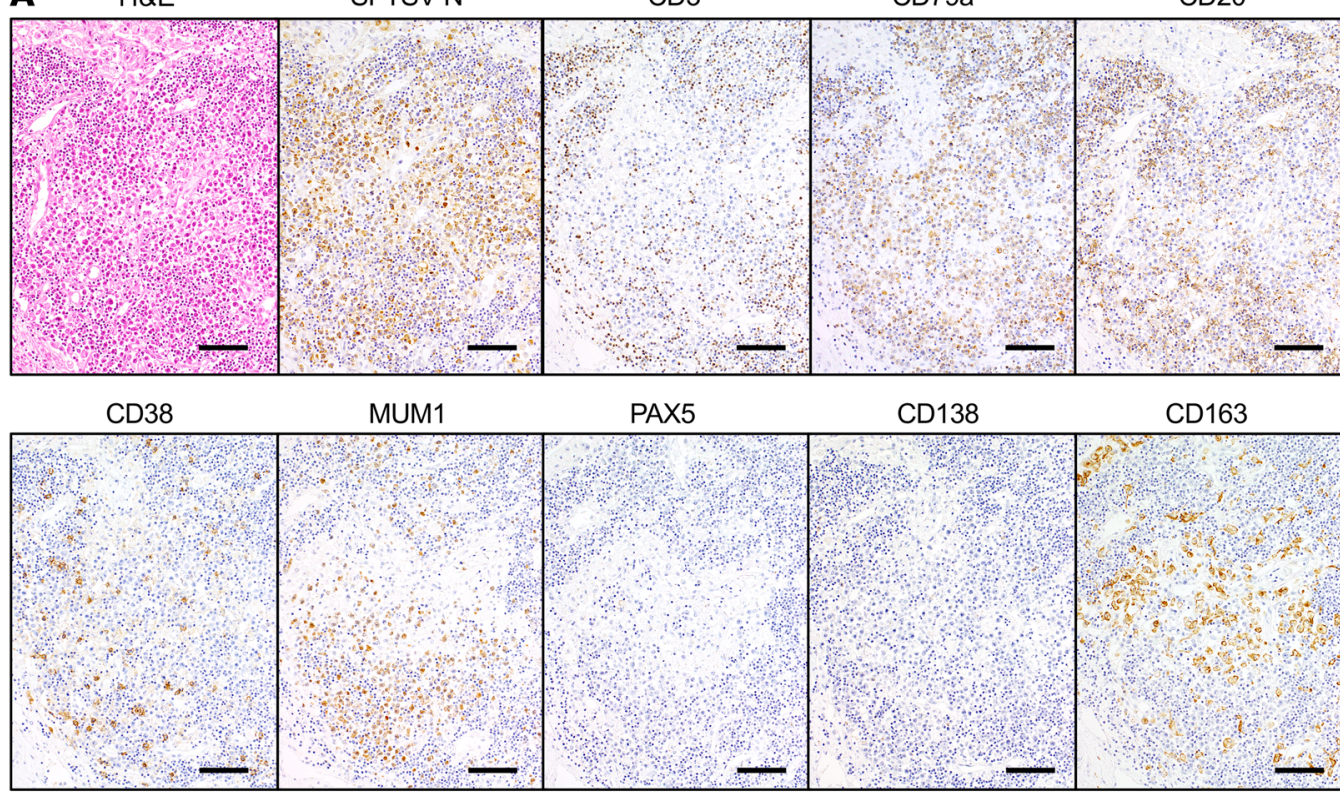

B
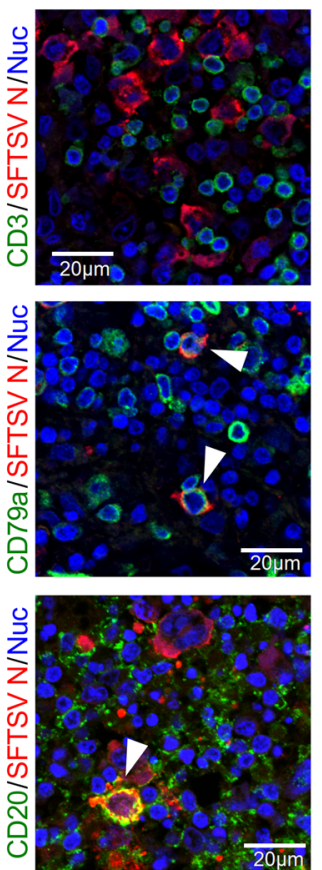
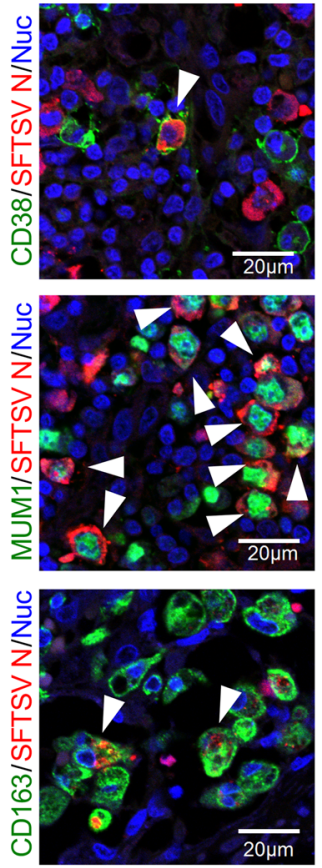

C
SFTSV antigenome/ SFTSV N/Nuc

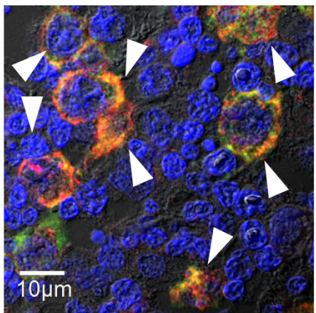

SFTSV antigenome/ MUM1/Nuc

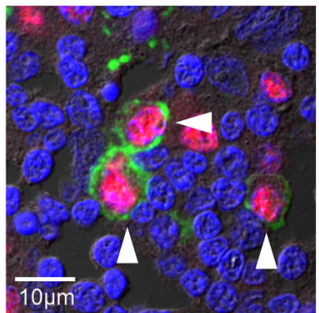

SFTSV antigenome/ CD20/Nuc

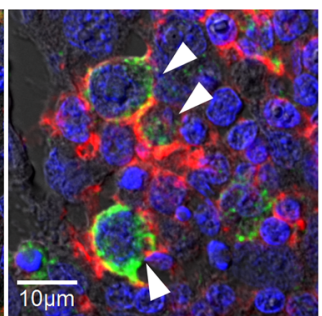

SFTSV antigenome/ CD163/Nuc

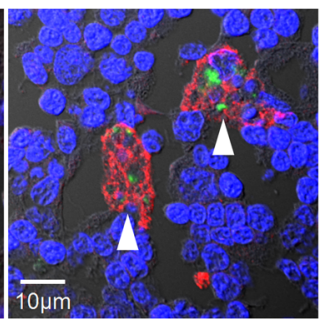

Figure 2. SFTSV-antigen+ cells in the lymph nodes of individuals who died because of SFTS are macrophages and $B$ celllineage lymphocytes. (A) IHC for immune-cell markers in an SFTSV+ lymph node. Scale bars: $100 \mu \mathrm{m}$. (B) Double IF staining for SFTSV N protein (red) and immune-cell markers (green). TO-PRO-3 nucleic acid staining (Nuc, blue) is also overlaid to produce the merged images. Arrowheads indicate colocalization of SFTSV and cell markers in the same cells. Scale bars: $20 \mu \mathrm{m}$. (C) FISH combined with IF staining for antigenomic SFTSV RNA (green) and SFTSV $\mathrm{N}$ protein (red, upper left) or immune-cell markers (red). Nuclear labeling by DAPI staining (Nuc, blue) and differential interference contrast (DIC) images were also overlaid to produce the merged images. Arrowheads indicate colocalization of antigenomic SFTSV RNA and IF staining in the same cells. Scale bars: $10 \mu \mathrm{m}$.
$\mathrm{XBP} 1$, which are nuclear transcription factors in post-germinal center B cells, and CD38, which is an activation marker expressed in post-germinal center B cells (38), were also positive in the same area (Figure 2A and Supplemental Figure 2). However, PAX-5, which is expressed in B cells from the pro-B cell stage to the plasma-cell stage, and CD138, which is highly upregulated in plasma cells, were not detected (Figure 2A). These observations indicated that post-germinal center B cells (prior to terminal differentiation to plasma cells) with some resemblance to a plasmablastic immunophenotype accumulated in the SFTSV-infected lymph nodes. Double immunofluorescence (IF) staining for SFTSV N and various cellular markers was performed to further characterize the SFTSV-infected cells in the lymph nodes, demonstrating that $\mathrm{CD}_{163^{+}}$macrophages stained for SFTSV N in their cytoplasm (Figure 2B). In addition, $\mathrm{SFTSV}^{+}$cells were mostly positive for CD79a, CD20, CD38, and MUM1 (Figure 2B). By contrast, SFTSV was not detected in $\mathrm{CD}^{+} \mathrm{T}$ cells. To determine whether SFTSV actively replicates in these cells, a combined fluorescence in situ hybridization (FISH) and IF staining assay, which enables simultaneous detection of antigenomic SFTSV RNA and proteins on a single sample slide, was performed. The assay demonstrated that SFTSV antigenomes were detected in $\mathrm{CD} 163^{+}$macrophages as well as $\mathrm{CD}_{2} \mathrm{O}^{+}$and $\mathrm{MUM} 1^{+} \mathrm{B}$ cells (Figure 2C), suggesting that SFTSV replicates in macrophages and B cells, and both of these cell types are susceptible to SFTSV. Notably, some SFTSV ${ }^{+}$cells lacked expression of MUM1 and CD38, but expressed CD20 (Sup- 
A

Liver

Adrenal

Intestine

Lung

Heart Capillary
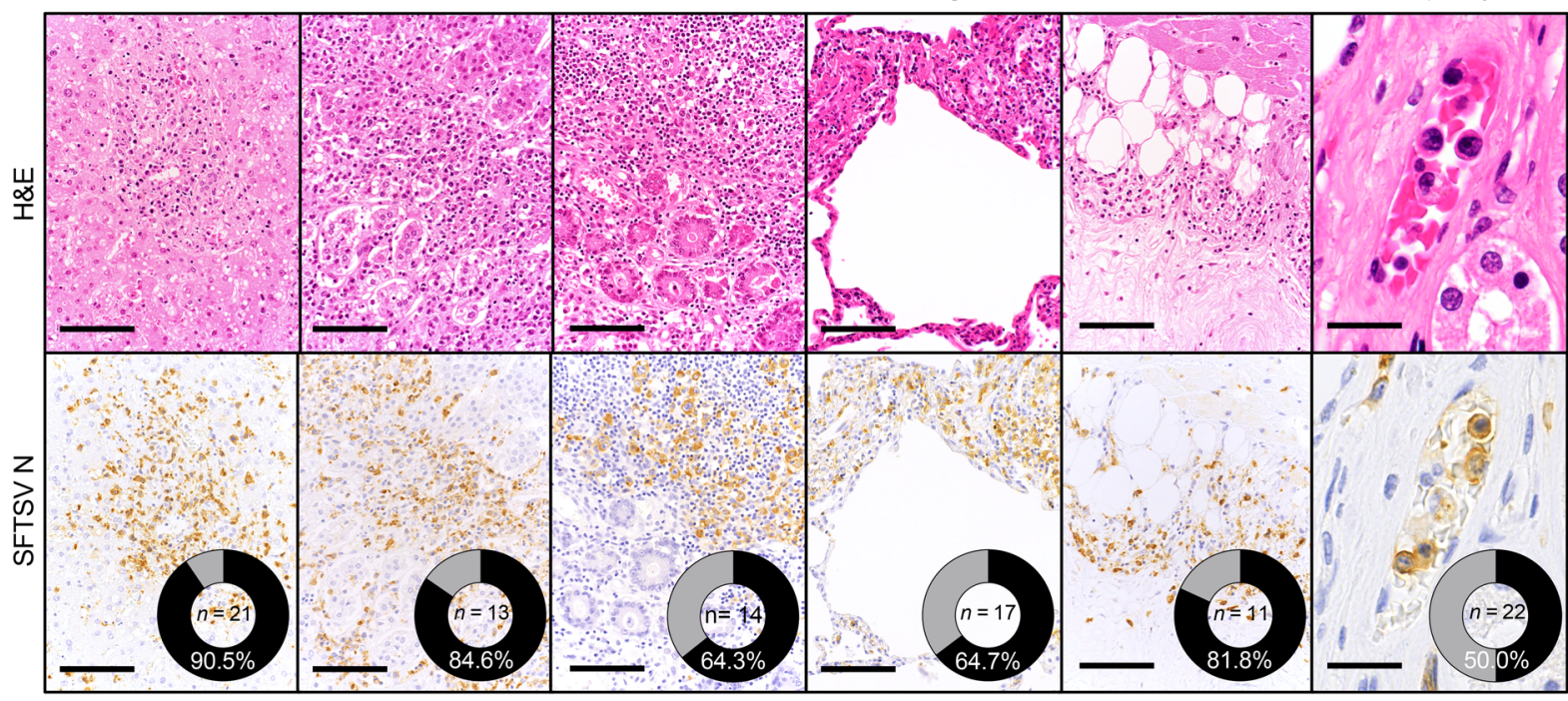

B

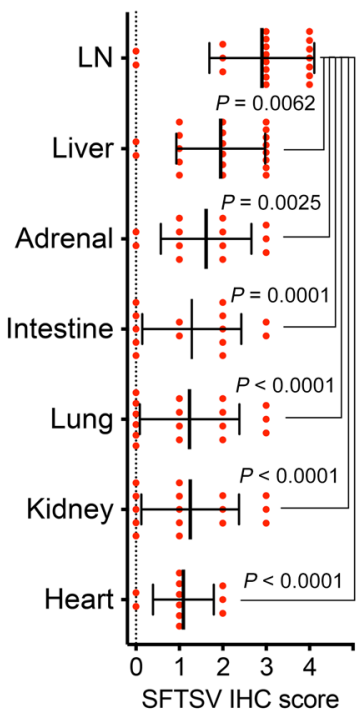

C

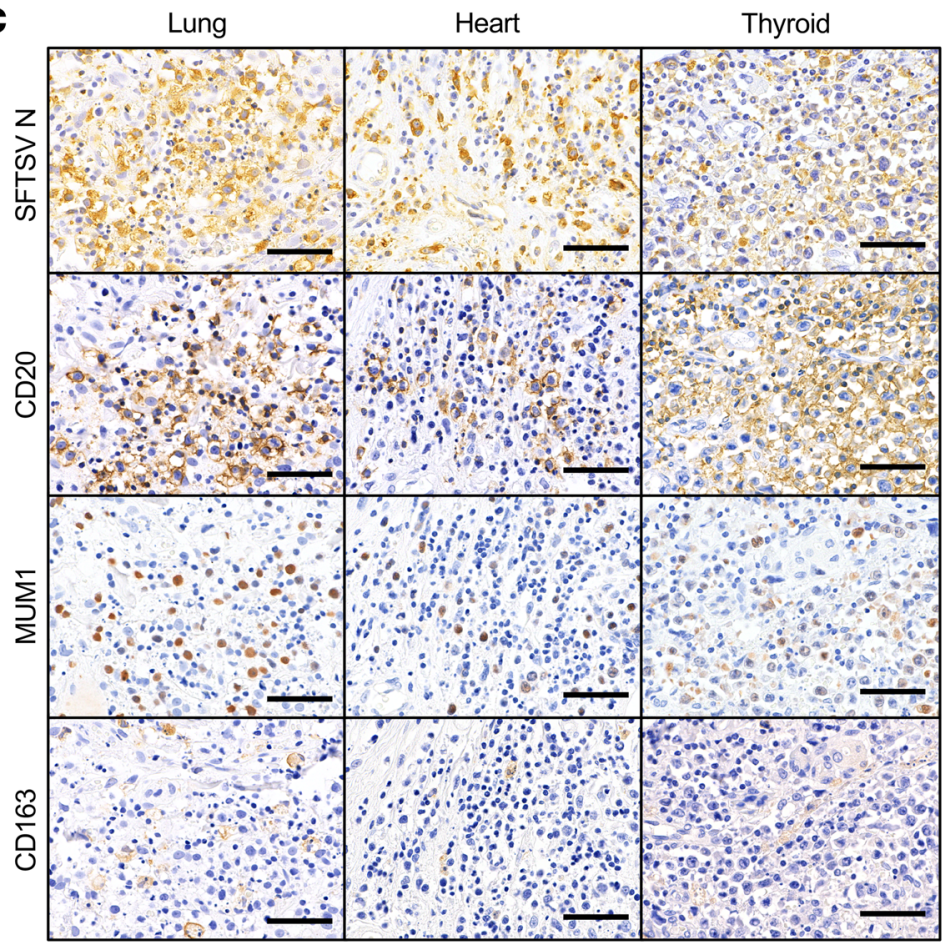

Figure 3. SFTSV-infected B cells infiltrate nonlymphoid organs in fatal SFTS. (A) Representative pathological images of the liver, adrenal gland, intestine, lung, heart, and tissue capillary (renal medulla) in individuals who died from SFTS. H\&E staining (upper panels) or IHC for SFTSV N protein (lower panels). Pie charts show the number of individuals whose tissues were analyzed, and the percentage in whose tissues SFTSV antigen was detected by IHC. Scale bars: $100 \mu \mathrm{m}$ or $20 \mu \mathrm{m}$ (capillary). (B) SFTSV IHC scores for tissue sections of lymph nodes (LNs; $n=20$ ), and nonlymphoid organs: liver ( $n=21$ ), adrenal gland $(n=13)$, intestine $(n=14)$, lung $(n=17)$, kidney $(n=16)$, and heart $(n=11)$. IHC scores for nonlymphoid organs were significantly lower than for LNs. Scatter plots also show mean \pm SD. The $P$ values were calculated with 1-way ANOVA followed by Holm-Sidak's multiple-comparisons test to make comparisons between the IHC scores of nonlymphoid organs and those of LNs. (C) IHC for SFTSV N, B cell (CD2O and MUM1), and macrophage (CD163) markers in serial tissue sections of nonlymphoid organs from individuals who died from SFTS. Regions with SFTSV+ cells had B cell infiltration. Scale bars: $50 \mu \mathrm{m}$.

plemental Figure 3), suggesting that SFTSV-susceptible lymphocytes belonged to the mature B cell lineage, but exhibited subtle phenotypic variation during differentiation to plasmablasts. In addition, several lymph nodes from multiple cases showed that the cells in the same area where numerous SFTSV-antigen ${ }^{+}$cells were detected, stained with cellular markers for B cells (CD20), but not macrophages (CD163) (Supplemental Figure 4). Furthermore, prominent hemophagocytosis by $\mathrm{CD} 163^{+}$macrophages with hemophagocytosis was identified abundantly in several areas within the lymph nodes where SFTSV-antigen ${ }^{+}$cells were not detected (Supplemental Figure 5). These observations indicated that B cells are dominant targets of SFTSV infection in lymph 


\section{A}

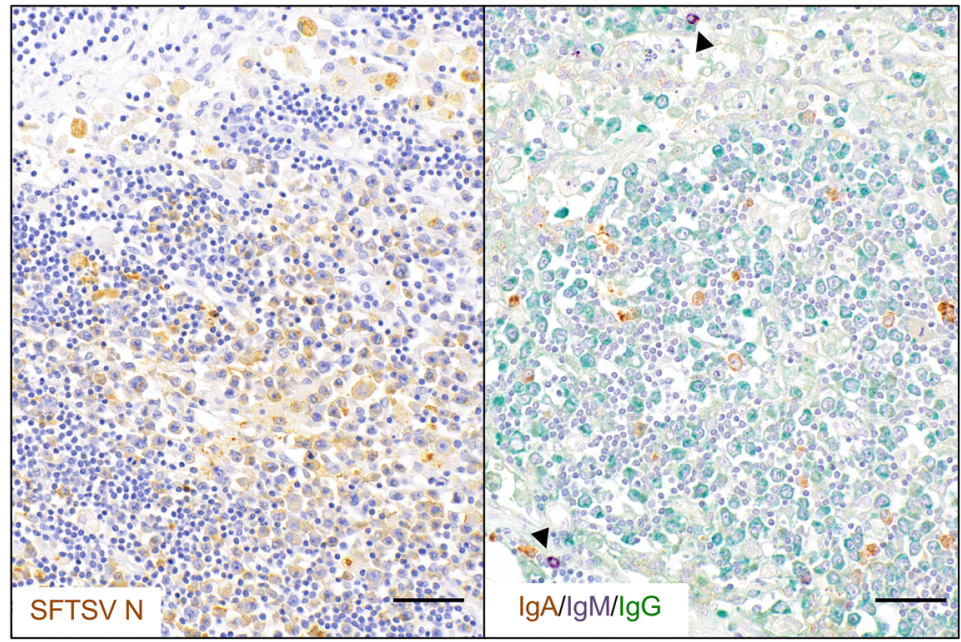

\section{B}

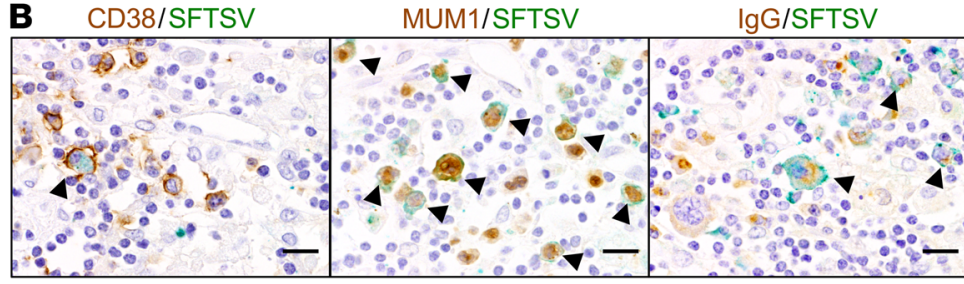

Figure 4. Class-switched IgG $\mathrm{C}^{+} \mathrm{B}$ cells are more susceptible to SFTSV infection than class-unswitched $\operatorname{lgM}^{+} B$ cells in lymphoid and nonlymphoid organs. (A) Representative images of chromogenic IHC staining for SFTSV N protein (left panel) or multiplex IHC staining for immunoglobulin heavy chains (IgA, brown; IgM, purple; IgG, green; right panel) on serial tissue sections of lymph node from an individual with fatal SFTS. The arrowhead in the right panel indicates an $\operatorname{lgM}^{+}$cell. Scale bars: $50 \mu \mathrm{m}$. (B) Representative images of chromogenic multiplex IHC staining for SFTSV (green) with activated lymphocyte marker (CD38, brown; left panel); plasmablast-lineage marker (MUM1, brown; middle panel); and IgG (brown; right panel). Arrowheads indicate colocalization of staining in cells. Scale bars: $10 \mu \mathrm{m}$. (C) Comparison between immunoglobulin heavy chain (IGH) mRNA levels (IGHG and IGHM) and SFTSV viral RNA levels in tissue sections of lymph nodes ( $n=58$, left panel) and nonlymphoid organs ( $n=36$, right panel) including the liver, adrenal gland, kidney, heart, and bladder, quantified by real-time RT-PCR. The SFTSV RNA load was positively correlated with the IGHG mRNA level in lymph node (Spearman's coefficient $=0.6027, P<0.0001$ ) and nonlymphoid organs (Spearman's coefficient $=0.8025, P<$ $0.0001)$. To compare regression lines, the $P$ values were calculated with nonlinear regression analysis with a straight-line model. ${ }^{* *} P=0.0045,{ }^{* * *} P<0.0001$
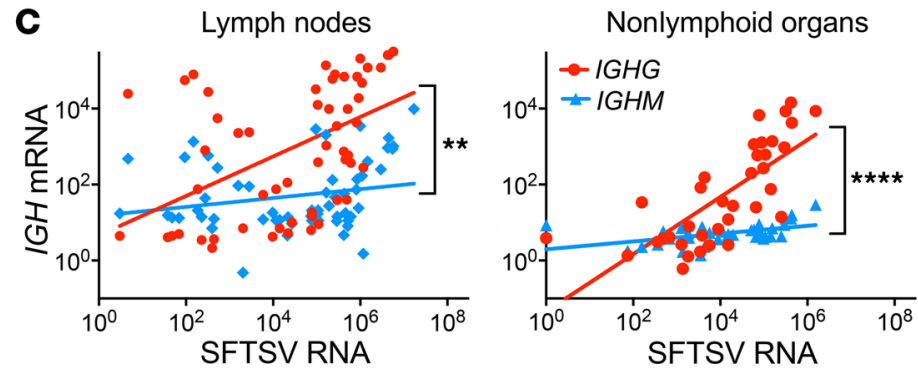

nodes at the time of death, and SFTSV infection in macrophages may not be the direct cause of hemophagocytosis, which is one of the important histopathological hallmarks of fatal SFTS. These IHC and FISH results demonstrated that SFTSV infected macrophages and mature $\mathrm{B}$ cells in the lymph nodes, and the majority of these B cells are activated and have a similar immunophenotype to that of plasmablasts.

Systemic dissemination of SFTSV-infected B cells in fatal SFTS. SFTSV-infected cells have been detected in multiple nonlymphoid organs, such as the liver, adrenal glands, intestine, heart, lungs, and kidneys in most examinations of fatal human SFTS (18, $34,37)$. Here, we analyzed, by IHC, the distribution of SFTSVinfected cells in nonlymphoid organs obtained postmortem from 22 individuals with fatal SFTS. Distribution of SFTSV antigen in the nonlymphoid organs was highly varied among these individuals. SFTSV ${ }^{+}$cells were detected in liver in approximately $90 \%$ of individuals, with detection rates in other nonlymphoid organs of 60\%-85\% (Figure 3A). SFTSV IHC scores (indicating the numbers of $\mathrm{SFTSV}^{+}$cells) were significantly lower in nonlymphoid organs than in lymph nodes (Figure 3B). These results supported our observation that SLOs, such as lymph nodes, are the primary target organs for human SFTSV infection. Notably, regions of the nonlymphoid organs containing $\mathrm{SFTSV}^{+}$cells were infiltrated with mononuclear cells, which were identified on hematoxylin and eosin (H\&E) staining (Figure 3A). In addition, the SFTSV ${ }^{+}$ cells were not parenchymal cells of these organs (Figure 3A). Furthermore, in nonlymphoid organs from half of the individuals, numerous SFTSV ${ }^{+}$mononuclear cells were identified in the capillaries (Figure 3A), suggesting that the SFTSV-infected mononuclear cells were not only resident in these nonlymphoid organs, but also disseminated into the systemic circulation of these individuals. IHC for markers of B cells or macrophages in tissue sections of nonlymphoid organs such as lung, heart, and thyroid detected numerous $\mathrm{CD}_{20} \mathrm{O}^{+}$and $\mathrm{MUM}^{+}$, but not $\mathrm{CD} 163^{+}$cells, in regions containing SFTSV ${ }^{+}$cells (Figure $3 \mathrm{C}$ ). In addition, $\mathrm{SFTSV}^{+}$mononuclear cells in the capillaries were stained with cellular markers for plasmablasts (MUM1 and CD38), but not macrophages (CD163) (Supplemental Figure 6). Interestingly, the SFTSV ${ }^{+}$cells in the capillaries lacked expression of CD20 (Supplemental Figure 6), which is associated with later stages of plasmablast-lineage differentiation compared with tissue-resident SFTSV-infected B cells. These results suggested that B cells at various stages of differentiation toward plasmablasts were disseminating into the nonlymphoid organs, serving as SFTSV replication sites. 
A

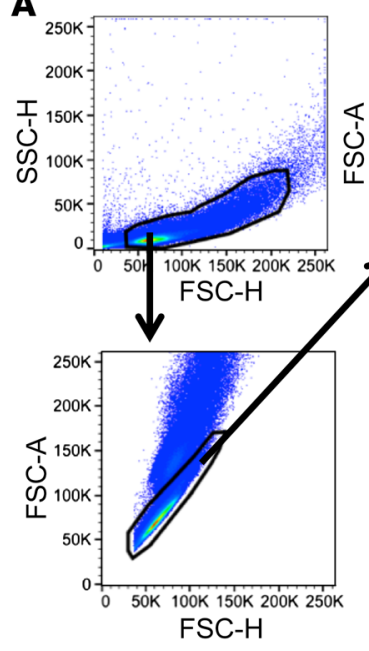

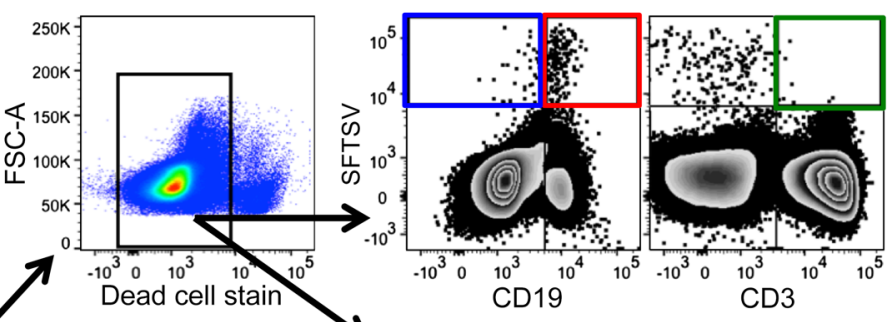
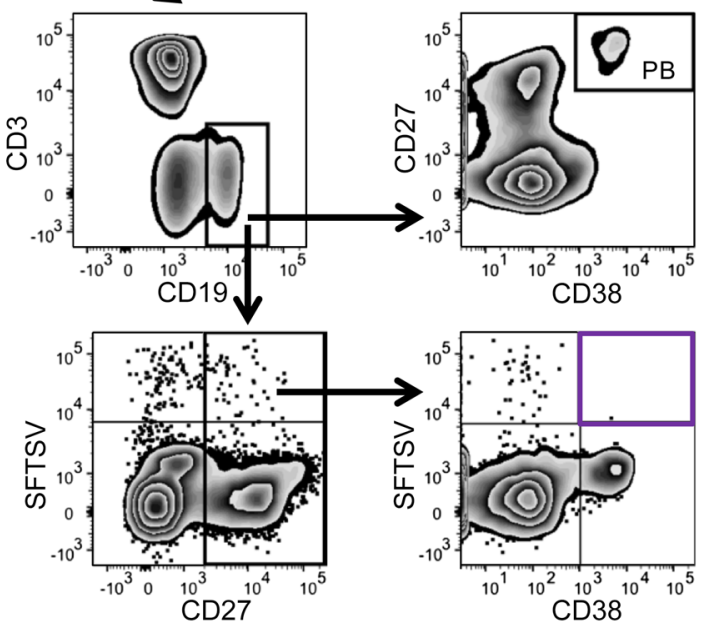

B

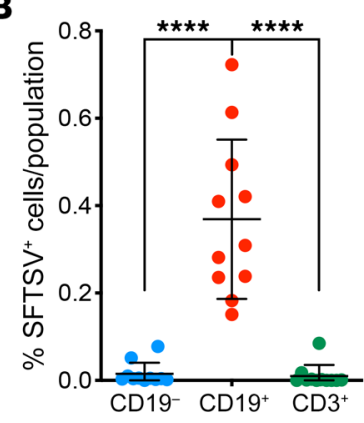

C

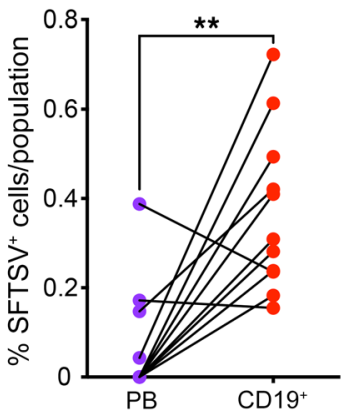

Figure 5. Peripheral-blood plasmablasts do not show higher susceptibility to SFTSV infection among peripheral-blood B cells in healthy adults. (A) Flow cytometry gating strategy to define lymphocyte subsets by CD3, CD19, CD27, and CD38 in SFTSV-inoculated PBMCs obtained from healthy donors ( $n=11$ ). The infectivity of SFTSV in each subset was determined by intracellular staining with DyLight 488-conjugated anti-SFTSV N antibody at 24 hours after inoculation. (B) Comparison of SFTSV infectivity among CD19- (blue), CD19+ (red), or CD3+ (green) lymphocytes in SFTSV-inoculated PBMCs. CD19+ B cells showed higher susceptibility to SFTSV than $\mathrm{CD}^{+} \mathrm{T}$ cells or CD19- non-B cells. Scatter plots also show mean $\pm \mathrm{SD}$. ${ }^{* * * *} P<0.0001$ (1-way ANOVA followed by HolmSidak's multiple-comparisons test). (C) Comparison of SFTSV infectivity between CD19+ B cells (red) and CD3-CD19+CD27 ${ }^{+}$CD38 ${ }^{+}$plasmablasts (PB, purple). The SFTSV-infected cells in CD19+ B cells were not biased to the PB fraction. Scatter plots also show paired lines $(n=11)$. ${ }^{* *} P=0.0024$ (paired $t$ test).

SFTSV antigen localizes to IgG $G^{+}$class-switched B cells in lymphoid and nonlymphoid organs. Individuals with fatal SFTS have impaired immunoglobulin gamma (IgG) antibody responses to SFTSV because of failure of B cell class switching (27). Normal plasmablasts are activated B cells in the germinal center that have undergone somatic hypermutation and class-switching recombination (38). These findings raise the possibility that SFTSV infection in $\mathrm{B}$ cells is closely related to the failure of $\mathrm{B}$ cell class switching. We assessed the expression of immunoglobulins in SFTSV-infected B cells in the lymph nodes by multicolor chromogenic IHC (Figure $4 \mathrm{~A})$. Numerous $\operatorname{IgG}^{+}$, but few $\operatorname{IgM}^{+}$cells, were present in lymph nodes that contained SFTSV ${ }^{+}$lymphocytes (Figure $4 \mathrm{~A}$ ). In these areas, $\mathrm{SFTSV}^{+}$cells coexpressing MUM1, CD38, or IgG were identified (Figure 4B), suggesting that SFTSV infected class-switched post-germinal center B cells. To confirm the tropism of SFTSV in B cell subsets, the levels of mRNA encoding gamma (IGHG) and $\mathrm{mu}(I G H M)$ heavy chains in lymph nodes or nonlymphoid organs with SFTSV infection were determined by quantitative reverse transcription PCR (qRT-PCR). The level of gamma heavy-chain mRNA was significantly correlated with the SFTSV RNA load in lymph nodes (Spearman's coefficient $=0.6027, P<0.0001$ ) and in nonlymphoid organs (Spearman's coefficient $=0.8025, P<$ 0.0001), whereas no significant correlation was found with the level of mu heavy-chain mRNA (Figure 4C). These results suggested that the number of $\mathrm{B}$ cells expressing $\operatorname{IgG}$ determined the extent of SFTSV replication in these tissues, and that the susceptibility to SFTSV was markedly higher in B cells expressing IgG than in B cells expressing IgM. Although the number of B cells in intact nonlymphoid organs is generally minimal, nonlymphoid organs from individuals who died as a result of SFTS showed prominent infiltration of mononuclear inflammatory cells, including B cells (Figure 3C). The strong correlation between IgG expression and SFTSV infection in the nonlymphoid organs (Figure 4C) implied that the $\mathrm{IgG}^{+} \mathrm{B}$ cells infiltrating into these organs were highly susceptible to SFTSV. Our results indicated that $\mathrm{IgG}^{+}$class-switched B cells might be the major targets for SFTSV infection in fatal SFTS.

SFTSV has limited ability to infect peripheral-blood plasmablasts obtained from healthy adults. To further examine the hypothesis that plasmablasts are susceptible to SFTSV infection, peripheral-blood mononuclear cells (PBMCs) obtained from healthy adult donors were assayed. Isolated PBMCs were inoculated with SFTSV for 24 hours at a multiplicity of infection (MOI) of 1 , and then analyzed by flow cytometry. Lymphocyte subsets were defined phenotypically by CD3, CD19, CD27, and CD38 cell-surface expression (Figure 5A). As a result, frequencies of cells stained by anti-SFTSV $\mathrm{N}$ antibody in $\mathrm{CD} 19^{+}$cells were low $(0.15 \%-0.74 \%)$. Thus, in order to confirm the specificity of SFTSV-antigen staining, infected or uninfected PBMCs were stained with an isotype control antibody or with an anti-SFTSV N antibody, respectively (Supplemental Figure 7). This assay also showed that SFTSV infectivity of 
A

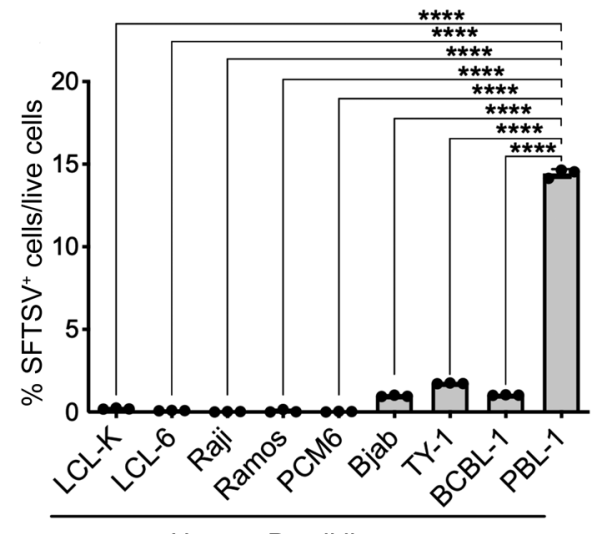

Human B cell lines
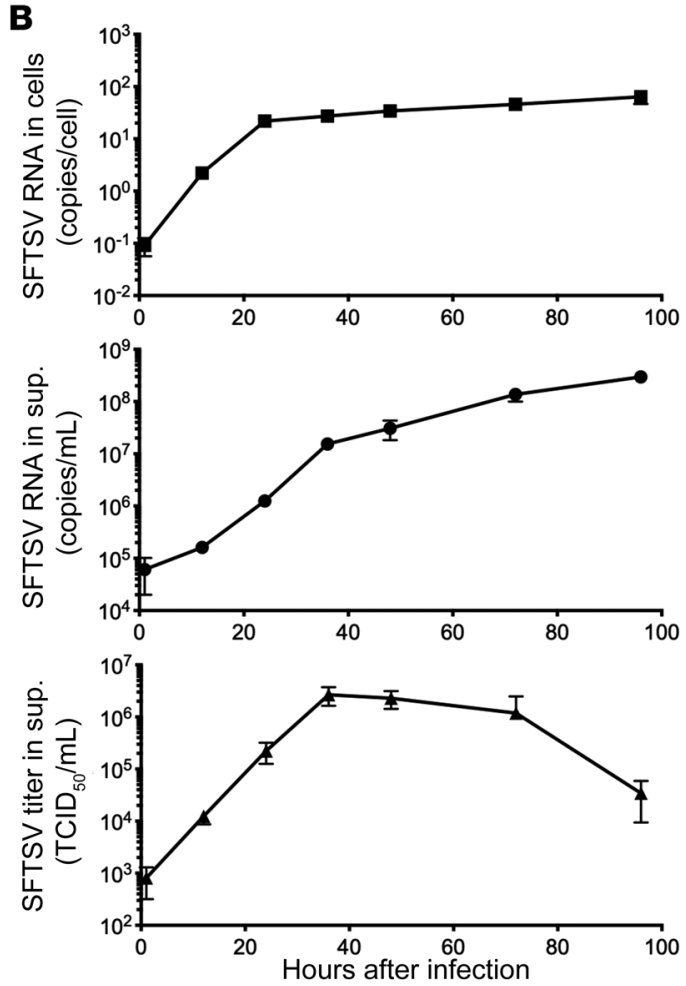

C

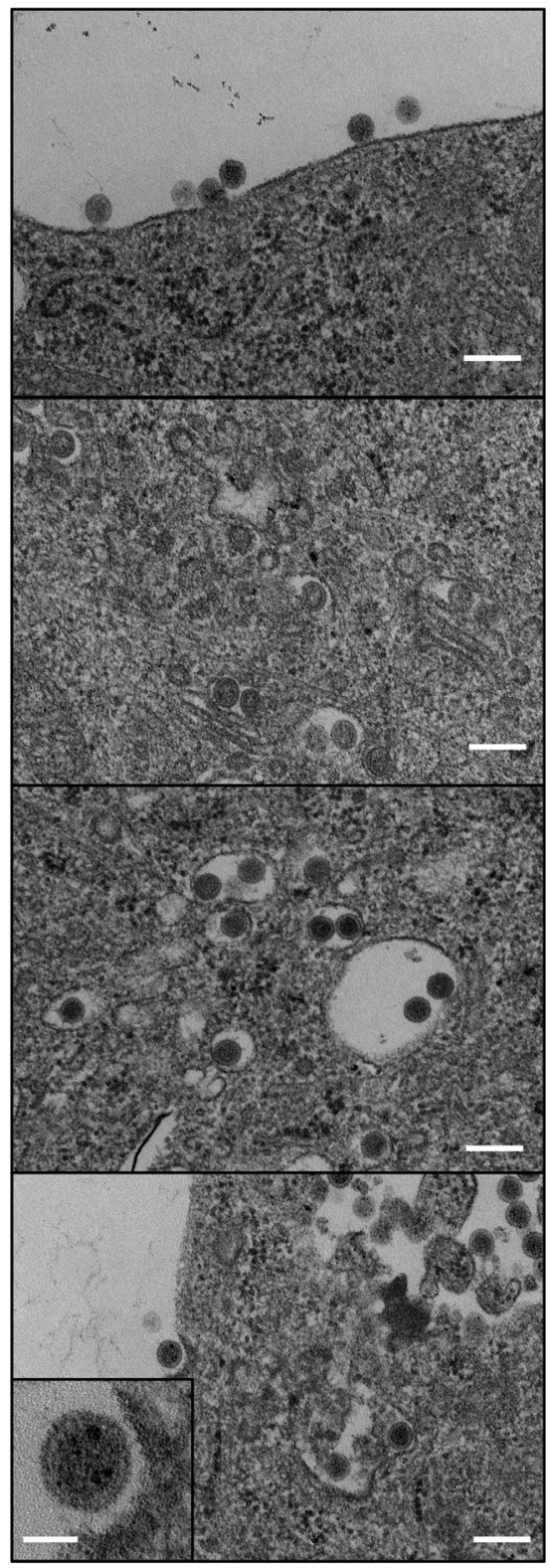

Figure 6. SFTSV propagates in the PBL-1 human plasmablastic lymphoma cell line. (A) SFTSV infectivity in human B cell lines LCL-K, LCL-6, Raji, Ramos, PCM6, Bjab, TY-1, BCBL-1, and $\mathrm{PBL}-1$. The cell lines were inoculated with SFTSV $(\mathrm{MOI}=10)$ and incubated for 24 hours. Infectivity in each cell line was determined by flow cytometry using DyLight 488-conjugated anti-SFTSV N protein antibody. Data are expressed as the mean \pm SD of 3 biological replicates. ${ }^{* * *} P$ $<0.0001$ (1-way ANOVA followed by Holm-Sidak's multiple-comparisons test to compare the SFTSV infectivity of PBL-1 with that of other B cell lines). (B) Kinetics of viral replication in PBL-1 cells was examined by serial sampling of cells and culture supernatants. SFTSV RNA loads in cells (upper panel) and culture supernatants (middle panel) at each time point were estimated by quantitative real-time RT-PCR. The quantity of infectious virions in culture supernatants at each time point was also estimated using Vero cells (SFTSV titer, lower panel). Data are expressed as the mean \pm SD of 3 biological replicates. (C) Electron microscopy of SFTSV-infected PBL-1 cells. Virus-like particles of 85 - to 90-nm diameter, with moderately to highly dense centers can be seen attached on the cell surface (upper panel), budding into the vacuoles (upper-middle panel), accumulating in intracellular vesicles (lowermiddle panel), and egressing from the vacuoles in the cytoplasm of PBL-1 (lower panel). Scale bars: 200 $\mathrm{nm}$ and $50 \mathrm{~nm}$ (inset).
$\mathrm{CD} 19^{+}$cells was significantly higher than that of $\mathrm{CD}^{+}$or $\mathrm{CD} 19^{-}$ cells (Figure 5B). Surprisingly, SFTSV infectivity of plasmablasts $\left(\mathrm{CD}^{-} \mathrm{CD} 19^{+} \mathrm{CD} 27^{+} \mathrm{CD} 38^{+}\right)$was significantly lower than that of $\mathrm{CD}^{+}{ }^{+} \mathrm{B}$ cells (Figure 5C), suggesting that SFTSV does not prefer infecting plasmablasts among the total B cell population circulating in the peripheral blood of healthy adults. Furthermore, it indicated that B cells undergoing plasmablast-lineage differentiation in SFTS patients possessed intrinsic properties different from that of peripheral-blood plasmablasts in healthy adults, despite its similarities in cell-surface-marker expression.

Human plasmablastic lymphoma cell line, $P B L-1$, is susceptible to SFTSV infection. Our results indicated differences in the relationship between SFTSV and plasmablasts from individuals with fatal SFTS and from healthy adults. In addition, lymphocyte cell lines, such as Jurkat and Raji, exhibit markedly lower susceptibility to SFTSV than mammalian adherent cell lines, such as Vero, Huh7, and COS7 (39). Notably, the B cell line of Raji cells has very low susceptibility to SFTSV (39). We therefore examined various human B cell lines for their susceptibility to SFTSV infection. Nine cell lines derived from various $\mathrm{B}$ cell malignancies were examined, because $\mathrm{B}$ cell malignancies are clonal tumors of mature and immature $\mathrm{B}$ cells at various stages of differentiation, and tend to mimic stages of normal B cell differentiation (40). The cell lines demonstrated different patterns of cell-surface-marker expression, reflecting the stage of differentiation of the original B cell malignancies (Supplemental Figure 8). PBL-1 cells, which are derived from a plasmablastic lymphoma (41), showed markedly higher susceptibility to SFTSV than other B cell lines (Figure 6A). In addition, infectious progeny virions egressed from SFTSV-inoculated PBL-1 cells into the culture supernatant (Figure 6B). However, the infectious virus titers of the culture supernatant decreased over time after 40 postinfection hours despite a continuous increase in SFTSV RNA 
A

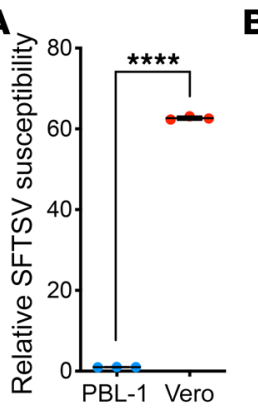

B

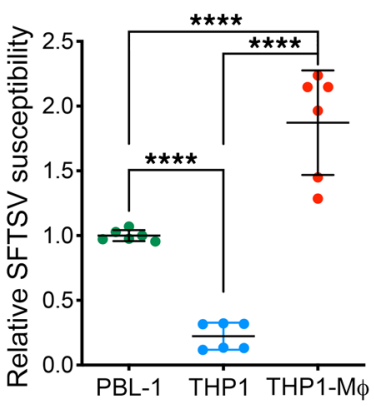

C

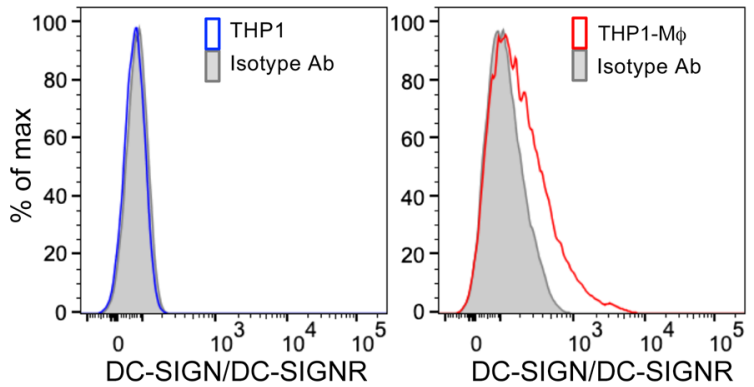

E
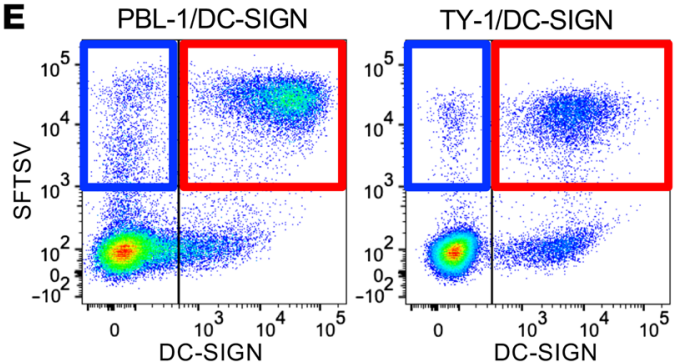

Raji/DC-SIGN

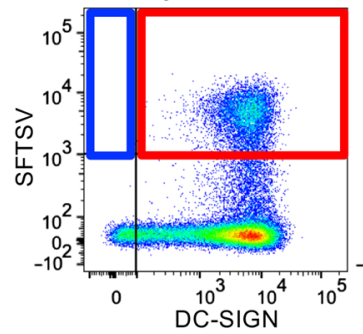

LCL-K/DC-SIGN

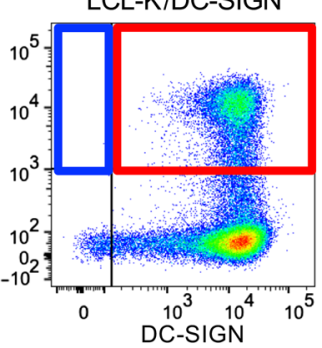

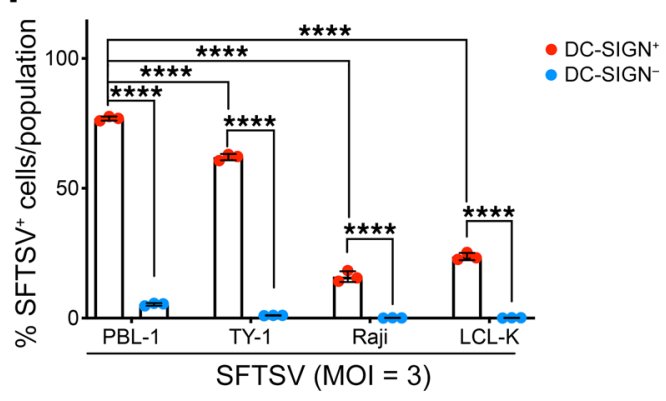

Figure 7. Expression of DC-SIGN enhances the susceptibility of PBL-1 cells to SFTSV infection. (A) Infectivity of SFTSV was compared in PBL-1 and Vero cells $(\mathrm{MOI}=1, n=3)$. Cells were inoculated with SFTSV, incubated for 24 hours, and then analyzed by flow cytometry with DyLight $488-$ labeled anti-SFTSV $\mathrm{N}$ protein antibody. Data are the mean \pm SD of biological replicates. ${ }^{* * *} P<0.0001$ (unpaired Student's $t$ test). (B) Infectivity of SFTSV was compared in PBL-1, THP-1, and human monocytic THP-1 cell-derived macrophages with PMA treatment (THP1-M $\phi)(\mathrm{MOI}=10, n=6)$. ${ }^{* * *} P<0.0001(1-$ way ANOVA followed by Holm-Sidak's multiple-comparisons test). (C) Cell-surface expression of DC-SIGN in THP-1 (left panel) or THP-1 cell-derived macrophages with PMA treatment (THP1-M $\phi$, right panel) was analyzed by flow cytometry with anti-DC-SIGN/DC-SIGNR antibody. (D) Cell-surface expression of DC-SIGN in PBL-1, TY-1, Raji, or LCL-K cells transfected with lentivector encoding fCD2 or DC-SICN, or in parent PBL-1 cells, was analyzed by flow cytometry with anti-DC-SIGN/DC-SIGNR antibody (left panel). SFTSV infectivity in PBL-1 cells, TY-1, Raji, or LCL-K cells expressing DC-SICN or fCD2, or in parental cells, was determined by flow cytometry using DyLight 488-labeled anti-SFTSV N antibody 24 hours after SFTSV inoculation (MOI = 3, right panel). (E) Relationship between expression of DC-SIGN on cell surface and SFTSV infectivity in PBL-1 cells, TY-1, Raji, or LCL-K cells expressing DC-SICN was examined by simultaneous staining with anti-DC-SIGN/DC-SIGNR antibody and DyLight 488-labeled anti-SFTSV N antibody. (F) Comparison of SFTSV infectivity between DC-SICN- (blue) and DC-SICN ${ }^{+}$(red) PBL-1, TY-1, Raji, and LCL-K cells demonstrated that expression of DC-SIGN significantly enhanced the susceptibility of these B cell lines to SFTSV infection, and infectivity of DC-SICN-expressing PBL-1 cells was significantly higher than that of DC-SIGN-expressing TY-1, Raji, or LCL-K cells. Data are the mean \pm SD of 3 biological replicates. ${ }^{* * *} P<0.0001$ (2-way ANOVA followed by Sidak's multiple-comparisons test). 
load in the culture supernatant (Figure 6B), which might reflect induction of cell death of SFTSV-infected PBL-1 cells during the late phase of infection. Furthermore, electron microscopic analysis identified numerous viral particles at various stages in the viral replication cycle, including cell-surface attachment, budding into intracellular organelles, accumulation in intracellular vesicles, and egress (Figure 6C), suggesting that PBL-1 cells could support the full SFTSV replication cycle. The origin of plasmablastic lymphoma is thought to be the plasmablast (41), and PBL-1 cells have an immunophenotype similar to that of terminally differentiated plasma cells $\left(\mathrm{CD} 138^{+} \mathrm{MUM}^{+}\right)$, suggesting that these cells are at an intermediate stage between the plasmablast and the plasma cell (Supplemental Figure 8). Moreover, primary effusion lymphoma cell lines (TY-1 and BCBL-1), whose postulated counterpart is a post-germinal center $B$ cell that has undergone plasmablastic differentiation, also showed moderate susceptibility to SFTSV, indicating that plasmablastic differentiation might determine susceptibility to SFTSV.

The expression of an exogenous viral entry mediator enhances the susceptibility of PBL-1 cells to SFTSV. Although PBL-1 cells were susceptible to SFTSV infection, it required a high viral MOI (Supplemental Figure 9), and the susceptibility was markedly lower (60-fold lower) than that of mammalian adherent Vero cells (Figure 7A). Pathological examinations demonstrated the existence of SFTSV-infected macrophages in addition to B cells undergoing differentiation toward plasmablasts in lymph nodes of fatal SFTS (Figure 2). Thus, SFTSV susceptibility of human monocytic THP-1 cell-derived macrophages (which were induced using phorbol 12-myristate 13-acetate [PMA]) were examined (42). As a result, SFTSV susceptibility of THP-1 cell-derived macrophages was significantly higher (2-fold higher) than that of PBL1 cells (Figure 7B). These observations indicated that PBL-1 lacks some factors (such as virus-entry mediators) that are necessary for efficient SFTSV replication. Notably, PBL-1 and other human B cell lines lacked cell-surface expression of the reported SFTSV receptors $(38,41,42)$ DC-SIGN, LSECtin, and NMHCIIa (Supplemental Figure 10). In contrast, THP-1 cell-derived macrophages expressed DC-SIGN, a C-type lectin and receptor for several viruses (including dengue virus) (Figure $7 \mathrm{C}$ ). To evaluate whether DC-SIGN improves the susceptibility of PBL-1 cells to SFTSV, PBL-1 cells were transfected with lentiviral vectors encoding DC-SIGN or feline CD2 (fCD2; as a negative control). Expression of DC-SIGN on the cell surface was confirmed (Figure 7D), and transfected cells were infected with SFTSV for 24 hours and analyzed by flow cytometry. Expression of DC-SIGN, but not fCD2, markedly increased SFTSV infection (Figure 7D). In addition, increase in SFTSV susceptibility by DC-SIGN expression was confirmed using other B cell lines: TY-1 (moderate susceptibility to SFTSV), Raji, and LCL-K (limited susceptibility to SFTSV) cells (Figure 7D). Furthermore, SFTSV infectivities of the DC-SIGN ${ }^{+}$ population within these DC-SIGN-expressing B cell lines were 15- to 200-fold higher than the DC-SIGN ${ }^{-}$population (Figure 7, $\mathrm{E}$ and $\mathrm{F}$ ), and the infectivity of DC-SIGN-expressing PBL-1 cells was significantly higher than that of DC-SIGN-expressing TY-1, Raji, or LCL-K cells (Figure 7, E and F). Notably, however, SFTSVinfected cells in the lymph nodes of individuals who died from SFTS did not express DC-SIGN (Supplemental Figure 11). These results suggest that expression of viral entry mediators other than DC-SIGN on the cell surface that are expressed in association with plasmablastic differentiation might regulate the susceptibility of plasmablasts to SFTSV infection in fatal SFTS.

\section{Discussion}

Despite serious public health concerns about SFTS, little is known about the underlying pathogenesis of the fatal disease. Here, we demonstrated that SFTSV-infected cells in lymph nodes, which are the primary target organs for SFTSV infection, are macrophages and class-switched B cells with a similar immunophenotype to that of plasmablasts. SFTS animal models and previous findings in other viral hemorrhagic fevers have shown that monocyte-lineage cells, including monocytes, dendritic cells, and macrophages, have a pivotal role in virus replication in vivo and in pathogenesis of the infection $(28,32)$. However, our pathological findings that SFTSV-infected cells in nonlymphoid organs also belonged to $\mathrm{B}$ cell lineages indicated that B cells, but not macrophages, might be involved in progression of human lethal SFTSV infection, particularly in the terminal stage of fatal SFTS. Notably, our analysis of autopsy tissues demonstrated a few cases in which the viral-antigen $^{+}$cells lacked expression of MUM1 and CD38 despite expression of CD20 (Supplemental Figure 3). Furthermore, SFTSVinfected B cells were also detected in the lumina of small vessels, particularly capillaries of nonlymphoid organs in half of the fatal SFTS cases (Figure 3A), and these SFTSV-infected cells in the capillaries showed an immunophenotype $\left(\mathrm{CD} 2 \mathrm{O}^{-} \mathrm{CD} 38^{+} \mathrm{MUM1}^{+}\right)$similar to that of B cells at a later stage of plasmablast-lineage differentiation (Supplemental Figure 6), which is comparable to those commonly observed in peripheral-blood plasmablasts in healthy individuals, and blood-derived memory B cells differentiating into plasmablasts (43). Plasmablasts are generally rare in the peripheral blood of healthy individuals, but expansions are observed briefly during responses to infection or vaccination $(43,44)$. In such situations, plasmablasts produce relatively high-affinity antibodies against the infecting pathogens or vaccine antigens, and, for viral infections, the magnitude of plasmablast proliferation closely correlates with viral load $(45,46)$. A fraction of the plasmablasts generated by the systemic immune response leave the SLOs and travel via the blood to the bone marrow $(43,44,47-50)$.

The low susceptibility to SFTSV of B cells and plasmablasts obtained from healthy individuals seems to contradict results showing that B cells in autopsy tissues are highly susceptible to SFTSV. Our previous results and those of other studies have demonstrated that plasmablasts appear transiently in the peripheral blood during the acute phase of SFTS $(27,51,52)$. In addition, the peripheral-blood plasmablasts proliferate to a greater extent in fatal SFTS than those in self-limiting SFTS, and the massive proliferated plasmablasts in fatal SFTS are dysfunctional (27). Detailed observations of peripheral blood from individuals with SFTS have revealed that effective humoral responses to SFTSV (specifically, IgG responses) are impaired in fatal SFTS because of a failure of B cell class switching (27). Our results showed that classswitched $\mathrm{IgG}^{+}$B cells were more susceptible to SFTSV infection than class-unswitched $\operatorname{IgM}^{+} \mathrm{B}$ cells in lymphoid and nonlymphoid organs (Figure 4C), which might have resulted in disturbance of normal plasmablast responses and aberrant induction of dysfunc- 
tional plasmablasts in these individuals. Accumulated evidence suggests that SFTSV-infected lymphocytes that were observed in the tissues of SFTS fatal cases belong to a mature B cell lineage, but exhibit phenotypes of B cells at various stages of differentiation to plasmablasts, and have unique characteristics that distinguish them from intact peripheral-blood B cells and plasmablasts obtained from healthy individuals.

Our previous observations on PBMCs obtained from 5 SFTS patients failed to detect SFTSV-infected cells in the peripheral blood (52). However, the existence of infected lymphocytes in the capillaries does not necessarily indicate that infected cells circulate in the peripheral blood. These observations are analogous to those commonly made in intravascular large B cell lymphoma (IVLB$\mathrm{CL}$ ), a rare type of non-Hodgkin lymphoma characterized by the selective growth of neoplastic cells within blood-vessel lumina. In IVLBCL, peripheral-blood lymphoma cells are detected in only $5 \%$ to $9 \%$ of patients, despite the intravascular growth pattern (53). In addition, the SFTSV-infected lymphocytes in the capillaries were detected in only half of the fatal SFTS cases we analyzed. Thus, considering the fact that $5 \%-40 \%$ of total SFTS cases are fatal, this phenomenon could only be observed in $2.5 \%-20 \%$ of total SFTS patients if it were unique for fatal SFTS cases. This may suggest that 5 patient cases were not enough to capture SFTSV-infected cells in the peripheral blood. Further studies including more SFTS patients are required to determine whether SFTSV-infected lymphocytes circulate in the peripheral blood or not.

In addition, we identified the susceptibility of the human plasmablastic lymphoma cell line PBL-1 to SFTSV replication, which supports the hypothesis that cell differentiation toward plasmablasts is a key determinant of the SFTSV susceptibility of $\mathrm{B}$ cells, and the pivotal roles of B cells in SFTS progression. However, the susceptibility of PBL-1 to SFTSV infection is limited compared with that of Vero cells that are commonly used for SFTSV propagation. Notably, expression of virus entry mediators such as DC-SIGN compensated for the low susceptibility to SFTSV of PBL-1 and other B cell lines. Of note, PBL-1 exhibited the highest susceptibility among all B cell lines regardless of DC-SIGN expression, suggesting that plasmablast-lineage differentiation provides the cellular machinery for virus replication, but not for efficient virus entry. Interestingly, SFTSV-infected cells in the lymph nodes of fatal SFTS showed lack of expression of DC-SIGN (Supplemental Figure 11), suggesting that DC-SIGN might play some roles in SFTSV infection of macrophages, but these DC-SIGN-expressing SFTSV-susceptible cells are not necessarily the major population among infected cells at the terminal stage of lethal SFTSV infection. Taken together, these observations implied that expression of host factors, except DC-SIGN, which are not usually expressed in intact B cells and plasmablasts, might regulate SFTSV susceptibility of B cells in the tissues of fatal SFTS cases, and these factors might be the crucial determinants of the fate of infected individuals. Further study will be required to elucidate these determinants of B cell susceptibility to SFTSV.

On the other hand, SFTSV-infected macrophages were also prominent in the primary target organs in fatal SFTS cases (Figure 2, B and C), and THP-1 cell-derived macrophages showed higher susceptibility to SFTSV infection than plasmablastic PBL-1 cells (Figure 7B), suggesting that macrophages may be playing important roles in virus replication. In addition, SFTSV-infected PBL-1 cells produce only a limited amount of infectious progeny virions in the late phase of infection, which prevent efficient propagation of virus, indicating that B cells alone may be not enough to provoke high-level virus replication, which is a clinical characteristic of fatal SFTS. Notably, prominent hemophagocytosis by $\mathrm{CD} 163^{+}$macrophages without SFTSV infection was observed in the lymph nodes of fatal SFTS (Supplemental Figure 5), suggesting that hemophagocytosis in SFTS patients could be induced by host responses following SFTSV infection, which might be configured by not only SFTSV-infected macrophages but also SFTSV-infected B cells. Taken together, our observations together with previous results indicate that each type of SFTSV-infected cell such as macrophages and plasmablast-lineage B cells may be playing different roles in SFTS pathogenesis, and initially infected macrophages might induce proliferation of SFTSV-susceptible plasmablast-lineage B cells, resulting in devastating SFTSV replication in the B cells and development of the catastrophic conditions of fatal SFTS. Further studies are needed to elucidate the interaction between macrophages and B cells in SFTS pathogenesis.

In summary, we have demonstrated that SFTSV infects macrophages and B cell-lineage lymphocytes in fatal SFTS, and that activated mature class-switched B cells undergoing plasmablastlineage differentiation in lymphoid and nonlymphoid organs are major targets for SFTSV at the end stage of lethal infection. SFTSV-infected B cells might have pivotal roles in clinical deterioration and fatal outcomes. In addition, our results also identify the potential for in vitro culture of SFTSV in PBL-1 cells, which bear remarkable resemblance to the targets of SFTSV in fatal SFTS. These cells may be of great clinical relevance for the study of SFTSV pathogenesis and the development of effective countermeasures.

\section{Methods}

Human specimens. Formalin-fixed, paraffin-embedded (FFPE) human tissues from 22 individuals who had died from SFTS were examined. SFTSV infection was confirmed by detection of viral RNA in serum or tissues by RT-PCR or real-time qRT-PCR. Autopsy tissue samples from the 22 individuals ( male $=50 \%$, mean age $=72 \pm 13$ years, mean days from onset to death $=11 \pm 1$ days) were originally submitted from 14 institutes to the Department of Pathology at the National Institute of Infectious Diseases for diagnostic consultation, and all the samples used in this study were residual tissues after the diagnostic purpose had been fulfilled. For SFTSV infection assay in PBMCs, PBMCs were isolated with Lymphoprep Tubes (Alere Technologies AS) following the manufacturer's instructions, suspended in RPMI 1640 medium (Gibco, Thermo Fisher Scientific) with $10 \%$ fetal bovine serum (FBS) (Biowest) and penicillin-streptomycin $(100 \mathrm{U} / \mathrm{mL}$ penicillin and 100 $\mu \mathrm{g} / \mathrm{mL}$ streptomycin; Gibco, Thermo Fisher Scientific), and immediately subjected to SFTSV infection.

Histopathological examination, IHC, and IF. All FFPE tissues were cut into 3- $\mu$ m-thick sections. One section from each tissue sample was stained using a standard H\&E procedure. Detection of the SFTSV N antigen was performed with a rabbit polyclonal antibody, as previously described (6). IHC for host-cell proteins was performed with antiCD3 (clone 2GV6, Ventana Medical Systems), and anti-BLIMP1 (clone EPR16655, Abcam) rabbit monoclonal antibodies, anti-DC-SIGN (clone 5D7, Abcam), anti-CD20 (clone L26, Leica Biosystems), anti- 
CD38 (clone SPC32, Leica Biosystems), anti-CD79a (clone JCB117, DAKO), anti-CD138 (clone MI15, DAKO), anti-MUM1 (clone MUM1p, DAKO), anti-PAX5 (clone 24/Pax-5, BD Biosciences), and anti-CD163 (clone 10D6, Leica Biosystems) mouse monoclonal antibodies, and anti-XBP1 catalog ab37152, Abcam) rabbit polyclonal antibody as the primary antibodies, and peroxidase-labeled polymer-conjugated antirabbit or anti-mouse immunoglobulin (EnVision/HRP; DAKO) as the secondary antibody. The CSA II system (Dako) was used for detection of MUM1 or PAX5. Immunostaining was visualized by 3,3'-diaminobenzidine tetrahydrochloride (DAB) staining. Hematoxylin (modified Mayer's) was used as a nuclear counterstain for IHC. The number of SFTSV-antigen ${ }^{+}$cells in each section was estimated from the number of antigen ${ }^{+}$cells in areas with highest cellularity of positive cells (no positive cells in the section $=$ score $0 ;<5$ positive cells per high-power field $[\mathrm{HPF}]=$ score $1 ;<50$ positive cells per $\mathrm{HPF}=$ score $2 ;<500$ positive cells per $\mathrm{HPF}=$ score $3 ; \geq 500$ positive cells per $\mathrm{HPF}=$ score 4 ). Representative IHC images for each score are shown in Supplemental Figure 1. Positive correlation between SFTSV IHC scores and SFTSV RNA load was identified (Supplemental Figure 1B). For IF staining, mouse monoclonal antibodies against cell type-specific marker proteins (CD20, CD38, CD79a, CD163, and MUM1) and rabbit polyclonal antibody against SFTSV N (or alternatively, anti-CD3 rabbit monoclonal and anti-SFTSV N mouse monoclonal [2D11] antibodies; see ref. 54) were used as primary antibodies. Alexa Fluor 488-conjugated anti-mouse IgG or anti-rabbit IgG, and Alexa Fluor 568-conjugated anti-rabbit IgG or anti-mouse IgG antibodies (Thermo Fisher Scientific) were used as secondary antibodies. The Tyramide Signal Amplification kit (Thermo Fisher Scientific) was used for detection of MUM1. TO-PRO-3 nucleic acid staining (Thermo Fisher Scientific) was used for detection of nuclei. Fluorescence images were visualized with confocal laser scanning microscopy (Olympus FV1000). Chromogenic multiplex IHC staining was performed with anti-IgG (clone A57H, Nichirei Biosciences), anti-IgM (clone R1/69, Nichirei Biosciences), anti-MUM-1, and anti-CD38 mouse monoclonal and anti-IgA (cata$\log$ 413581, Nichirei Biosciences) rabbit polyclonal primary antibodies, respectively. DAB Chromogen Kit (Biocare Medical), VECTOR VIP Peroxidase (HRP) Substrate Kit (Vector Laboratories), and Vina Green Chromogen Kit (Biocare Medical) were used as chromogens for horseradish peroxidase visualization. Following the first staining for each cell marker using the polymer-based detection system (Biocare Medical) with DAB, a denaturing step was carried out by hydrolytic autoclaving in citrate buffer ( $\mathrm{pH}$ 6.0) for 10 minutes at $121^{\circ} \mathrm{C}$. A second staining was then performed with VECTOR VIP, followed by the denaturing step again. A final staining was performed for viral antigens with Vina Green. Nuclei were counterstained with hematoxylin.

FISH combined with IF. SFTSV has a trisegmented, single-stranded RNA genome with negative ( $\mathrm{L}$ and $\mathrm{M}$ segments) or ambisense (S segment) polarity (4). The S segment encodes the nucleocapsid protein $(\mathrm{N})$ in antisense orientation and the nonstructural protein (NSs) in sense orientation, which means that positive-sense RNA for the $\mathrm{N}$ gene is antigenomic RNA, and the detection of the positive-sense antigenomic RNA in the cells demonstrates SFTSV genome replication in these cells. To detect SFTSV antigenome in FFPE tissues, ISH was performed using the RNAScope Multiplex Fluorescent V2 Assay (Advanced Cell Diagnostics) with TSA Plus fluorescein (PerkinElmer) according to the manufacturers' instructions. Briefly, ZZ probe pairs with channel C1 targeting the antigenomic SFTSV N gene were designed and synthesized by Advanced Cell Diagnostics (RNAscope Probe V-SFTSV-NP, catalog 449891). FFPE tissue sections were exposed to ISH target probes and incubated at $40^{\circ} \mathrm{C}$ in a hybridization oven for 2 hours. After rinsing, the ISH signal was amplified using company-provided preamplifier and amplifier conjugated to fluorescent dye. The RNAScope assay was followed by standard IF staining with mouse monoclonal antibodies against cell type-specific marker proteins (CD20, CD163, and MUM1) and rabbit polyclonal antibody against SFTSV N antibodies as primary antibodies. Alexa Fluor 568conjugated anti-rabbit IgG or anti-mouse IgG antibodies (Thermo Fisher Scientific) were used as secondary antibodies. Sections were counterstained with 4',6-diamidino-2-phenylindole (DAPI) (Thermo Fisher Scientific), mounted, and stored at $4^{\circ} \mathrm{C}$ until image analysis.

$R N A$ extraction and $q R T-P C R$. RNA was extracted with the Pure Link FFPE RNA isolation kit (Thermo Fisher Scientific), and qRT-PCR was performed using the QuantiTect Multiplex RT-PCR Kit (Qiagen) and an Mx3005P system (Agilent), according to the manufacturers' protocols. The $\mathrm{N}$ region within the $\mathrm{S}$ segment of the SFTSV genome was amplified. The amount of human ACTB mRNA in the DNase-treated RNA extracted from each section was also determined and used as an internal reference for normalization. The following primers and labeled probes were used:SFTSV, forward 5'-CCCTGATGCCTTGACGATCT-3' and reverse 5'-TGATTGGGTGAGGGACACAAAGTT-3'; probe 5'-(FAM)-TTGCCTCGAGTCAGGGCAAAGACAA-(TAMRA)-3'; human ACTB mRNA, forward 5'-TGAGCGCGGCTACAGCTT-3' and reverse 5'-TCCTTAATGTCACGCACGATTT-3'; probe 5'-(FAM)-ACCACCACGGCCGAGCGG-(TAMRA)-3'; human gamma heavy chain (IGHG) mRNA, forward 5'-GGCAGCCGGAGAACAAC-3' and reverse 5'-CCACGGTKAGCYTGCTGTAGA-3'; probe 5'-(FAM)-TGCTGGACTCCGACGGCTCCTT-(BHQ1)-3'; human mu heavy chain (IGHM) mRNA, forward 5'-ATGGCGAAGCTGTGAAAACC-3' and reverse 5'-TTCCAGTCATCCTCGCAGATG-3'; and probe 5'-(CY5)-ACACCAACATCTCCGAGAGCCACCC-(BHQ3)-3'.

Viruses and cells. All cells were cultured at $37^{\circ} \mathrm{C}$ in an atmosphere containing $5 \% \mathrm{CO}_{2}$. PBL-1 (41) and PCM6 (a human myeloma cell line, Riken Bioresource Center, RCB1460; ref. 55) cells were maintained in RPMI 1640 medium supplemented with 5 ng/mL IL-6 (PeproTech), 10\% FBS, and penicillin-streptomycin (Thermo Fisher Scientific). Other human B cell lines used were 2 Epstein-Barr virus-transformed lymphoblastoid cell lines (LCL-K and LCL-6, established in our laboratory), 3 Burkitt lymphoma cell lines (Raji, Ramos, and Bjab, provided by Department of Pathology, Institute of Medical Science, the University of Tokyo), and 2 primary effusion lymphoma cell lines (TY-1 and BCBL-1; refs. 56, 57) were cultured in RPMI 1640 medium with $10 \%$ FBS and penicillin-streptomycin. THP-1 cells were maintained in RPMI 1640 medium supplemented with 10\% FBS, penicillin-streptomycin, $1 \mathrm{mM}$ sodium pyruvate (Thermo Fisher Scientific), $0.1 \mathrm{mM}$ nonessential amino acids (Thermo Fisher Scientific), and $55 \mu \mathrm{M}$ 2-mercaptoethanol (Thermo Fisher Scientific). THP-1 cells (ATCC, TIB-202) were differentiated to macrophages at a density of $2 \times 10^{5}$ cells/mL in HydroCell 6 multi-well plates (CellSeed Inc.), treated with 100 nM PMA (Sigma-Aldrich) for 72 hours (42), washed, suspended in culture medium without PMA, and then subjected to SFTSV infectious assay. Vero cells (JCRB Cell Bank, JCRB9013) were cultured in Dulbecco's modified Eagle's medium (DMEM) (Sigma-Aldrich) supplemented with 10\% FBS and penicillin-streptomycin. Virus-inoculated cells were maintained in DMEM containing 2\% FBS and penicillin-strep- 
tomycin. SFTSV was propagated in Vero cells. The HB29 strain was used, which was isolated from a Chinese patient with SFTS (1). Titration of SFTSV was performed by plaque assay with Vero cells, as previously described (58). Confluent monolayers of Vero cells in 12-well plates were inoculated with serially diluted SFTSV for 1 hour at $37^{\circ} \mathrm{C}$. After washing with PBS, the cells were cultured with DMEM containing $2 \%$ FBS and $1 \%$ agarose for 3 days, and then a mixture of neutral red $(0.005 \%$ final concentration) and methylene blue $(0.005 \%$ final concentration) was added to the culture medium. The next day, visualized plaques were counted.

Virus infection. To examine SFTSV infectivity in PBMCs, human B cell lines, or Vero cells, the cells were infected with SFTSV at an MOI of 1 (PBMCs), 10 (human B-cell lines and THP-1 cells), or 1 (Vero cells). Cells were cultured for 24 hours. SFTSV infectivity was determined by staining with antibody against SFTSV N, with analysis by flow cytometry. To examine the dose dependency of SFTSV infectivity, PBL-1 cells were infected with SFTSV (at MOI of 1, 10, or 100), and then cultured for 24 hours. Infectivity was determined by flow cytometry. To examine the effect of expression of DC-SIGN on susceptibility to SFTSV, PBL-1, TY-1, Raji, and LCL-K cells stably expressing DC-SIGN or fCD2 were established using recombinant lentiviral vectors, as described previously (39). These cells were infected with SFTSV at MOI $=3$. SFTSV infectivity was determined by flow cytometry after 24 hours of culture.

Flow cytometry. For cell-surface staining, cells were incubated with Human FcR Blocking Reagent (Miltenyi Biotec) for 15 minutes prior to staining with fluorescent dye-conjugated antibodies against cell-surface markers. Mouse monoclonal antibodies against CD3 (PE-conjugated, clone HIT3a), CD19 (APC-conjugated, clone HIB19), CD38 (PE-conjugated or Alexa Fluor 700-conjugated, clone HIT2), CD24 (APC-conjugated, clone ML5), and CD20 (PE-conjugated, clone $2 \mathrm{H7}$ ) were obtained from BioLegend. FITC-conjugated antiCD138 (rat clone 281-2, BD Biosciences) rat monoclonal antibody, and APC-conjugated anti-CD27 (clone O323, eBioscience, Thermo Fisher Scientific), PE-conjugated anti-LSECtin (catalog sc-65478PE, Santa Cruz Biotechnology), and PE-conjugated anti-DC-SIGN+DCSIGNR (clone 120612, R\&D Systems) mouse monoclonal antibodies were also used for cell-surface staining. To examine the expression of MUM1 or non-muscle myosin IIA heavy chain (NMHCIIa), cells were fixed and permeabilized using BD Cytofix/Cytoperm Fixation Permeabilization Solution Kit (BD Biosciences Pharmingen), and incubated with Alexa Fluor 647-conjugated IRF4 rat monoclonal antibody (clone IRF4.3E4, BioLegend) or anti-NMHCIIa rabbit monoclonal antibody (clone EPR8965, Abcam), respectively. For detection of NMHCIIa, cells were then incubated with DyLight 488-conjugated donkey anti-rabbit IgG antibody (catalog 406404, BioLegend). Expression of NMHCIIa on the cell surface was also examined by staining the unfixed cells with the same antibodies. For detection of SFTSV-infected cells, the fixed and permeabilized cells were stained with DyLight 488-conjugated anti-SFTSV N monoclonal (2D11 or 9D3) antibody (54), which was prepared with the DyLight 488 Microscale Antibody Labeling Kit (Thermo Fisher Scientific) according to the manufacturer's instructions. Alexa Fluor 488-conjugated mouse IgG1, $\kappa$ isotype control antibody (clone MOPC-21, BioLegend) was used as an isotype control antibody. LIVE/DEAD Fixable Aqua Dead Cell Stain kit (Thermo Fisher Scientific) or eBioscience Fixable Viability Dye eFluor 450 (Thermo Fisher Scientific) was used for exclusion of dead cells according to the manufacturer's instructions. Cells were washed and analyzed by flow cytometry (FACSCanto II, BD Biosciences). Data were analyzed with FlowJo software (FlowJo, LLC).

Viral growth assay in PBL-1 cells. PBL-1 cells were infected with virus at MOI $=10$ for 1 hour. The cells were washed with PBS and cultured with RPMI 1640 containing $5 \mathrm{ng} / \mathrm{mL}$ IL-6, 10\% FBS, and penicillin-streptomycin. Cells and culture supernatants were harvested 1 hour after the start of cell culture and at 12, 24, 36, 48, 72, and 96 hours after inoculation. RNA was purified from each sample with TRIzol (Thermo Fisher Scientific) and a Direct-zol RNA microPrep kit (Zymo Research), according to the manufacturers' protocols. SFTSV RNA loads were quantified by qRT-PCR. RNA load per cell was calculated with the human GAPDH mRNA copy number as an internal control to estimate the cell number for each extracted RNA. The primers and labeled probes used for amplification of human GAPDH mRNA were previously described (59). The titer of infectious virions in the culture supernatant at each time point was calculated by counting the average $\mathrm{TCID}_{50}$ of 3 replicates according to the Reed-Muench method. In brief, 10 -fold serial dilutions of the culture supernatants were inoculated onto Vero cells in a 96-well culture plate and incubated for 3 days. The cytopathic effect observed in the virus-containing wells after 1 blind passage was evaluated under a microscope and the $\mathrm{TCID}_{50}$ calculated.

Electron microscopy. PBL-1 cells were infected with SFTSV at MOI = 10 and incubated for 2 days. Cells were pelleted by centrifugation, fixed with $2.5 \%$ glutaraldehyde and $2 \%$ paraformaldehyde in $0.1 \mathrm{M}$ phosphate buffer ( $\mathrm{pH} 7.4$ ) for 2 hours at room temperature, postfixed in $1 \%$ osmium tetroxide, and embedded in Epon. Ultrathin sections were stained with uranyl acetate and lead citrate, and observed with a transmission electron microscope (HT7700, Hitachi High Technologies) at $80 \mathrm{kV}$.

Statistics. All statistical analyses, graphical representations, and correlation and linear regression analyses were performed with the Prism statistical software package (version 6.0, GraphPad Software). The paired or unpaired 2-tailed Student's $t$ test, Wilcoxon's matchedpairs signed-rank test, 1-way ANOVA followed by Holm-Sidak's multiple-comparisons test, the Kruskal-Wallis test followed by Dunn's multiple-comparison test, Spearman's rank-correlation coefficients, nonlinear regression analysis with a straight-line model, or 2-way ANOVA followed by Sidak's multiple-comparisons test was used for analysis of each data set as indicated in the figure legends. A $P$ value less than 0.05 was considered significant.

Study approval. This study was approved by the medical research ethics committee of the National Institute of Infectious Diseases for the use of human subjects, Tokyo, Japan (no. 427, 517, and 821). This study was performed on postmortem human tissues procured after the participant's guardians provided written informed consent for an autopsy and research use of surplus tissue from the autopsy. For SFTSV-infection assay in PBMCs, peripheral-blood samples were collected from 11 healthy adult donors after provision of written informed consent from all participants.

\section{Author contributions}

TS and $\mathrm{HH}$ designed the research study. TS conceived and designed the experiments. TS and $\mathrm{HH}$ collected human specimens. TS, YS, HK, KT, NN, and HH conducted histopathological examinations. YS conducted H\&E staining, IHC, and IF experiments. NN conducted FISH experiments. TS acquired microscopic images. TS and KS conducted molecular pathological experiments and analyzed data. TS, KS, YW, TA, and M. Shimojima conducted 
in vitro experiments using PBMCs and cell lines. MK conducted EM analysis and acquired EM images. SM, SF, TY, M. Shimojima, and M. Saijo provided reagents for histopathological and virological assays. TS wrote the original draft of the manuscript. All authors contributed to reviewing and editing the manuscript. SM and M. Saijo supervised the study and supported analyses. TS and $\mathrm{HH}$ supervised the study.

\section{Acknowledgments}

Chinese SFTSV strain HB29 was a gift from Dexin Li and Mifang Liang at the National Institute for Viral Disease Control and Prevention, Chinese Center for Disease Control and Prevention. We thank Akiko Sataka (National Institute of Infectious Diseases) for technical support with in vitro experiments and Keita Matsuno (Hokkaido University) for his valuable suggestions about FISH experiments and virus infectious assays using
THP-1 cells. We would also like to thank all the clinicians and the pathologists who submitted specimens to the Department of Pathology, National Institute of Infectious Diseases, for pathogen testing. The work was supported in part by the Emerging/ Re-emerging Infectious Diseases Project of Japan, from the Japan Agency for Medical Research and Development, AMED grants JP18fk0108002, JP19fk0108104, and JP19fk0108081, and J-PRIDE from AMED grant JP19fm0208002. The funders had no role in study design, data collection and analysis, decision to publish, or preparation of the manuscript.

Address correspondence to: Tadaki Suzuki or Hideki Hasegawa, Department of Pathology, National Institute of Infectious Diseases, Toyama 1-23-1, Shinjuku, Tokyo 162-8640, Japan. Phone: 81.3.5285.1111;Email:tksuzuki@nih.go.jp(TS);Email:hasegawa@ nih.go.jp (HS).
1. Yu XJ, et al. Fever with thrombocytopenia associated with a novel bunyavirus in China. $N$ Engl J Med. 2011;364(16):1523-1532.

2. McMullan LK, et al. A new phlebovirus associated with severe febrile illness in Missouri. $N$ EnglJ Med. 2012;367(9):834-841.

3. Kim KH, et al. Severe fever with thrombocytopenia syndrome, South Korea, 2012. Emerging Infect Dis. 2013;19(11):1892-1894.

4. Liu S, et al. Systematic review of severe fever with thrombocytopenia syndrome: virology, epidemiology, and clinical characteristics. Rev Med Virol. 2014;24(2):90-102.

5. Liu Q, He B, Huang SY, Wei F, Zhu XQ. Severe fever with thrombocytopenia syndrome, an emerging tick-borne zoonosis. Lancet Infect Dis. 2014;14(8):763-772

6. Takahashi $\mathrm{T}$, et al. The first identification and retrospective study of severe fever with thrombocytopenia syndrome in Japan. J Infect Dis. 2014;209(6):816-827.

7. Yoshikawa T, et al. Phylogenetic and geographic relationships of severe fever with thrombocytopenia syndrome virus in China, South Korea, and Japan. J Infect Dis. 2015;212(6):889-898.

8. Saijo M. Pathophysiology of severe fever with thrombocytopenia syndrome and development of specific antiviral therapy. JInfect Chemother. 2018;24(10):773-781.

9. Yun SM, et al. Severe fever with thrombocytopenia syndrome virus in ticks collected from humans, South Korea, 2013. Emerging Infect Dis. 2014;20(8):1358-1361.

10. Gai ZT, et al. Clinical progress and risk factors for death in severe fever with thrombocytopenia syndrome patients. JInfect Dis. 2012;206(7):1095-1102.

11. Zhang YZ, et al. Hemorrhagic fever caused by a novel Bunyavirus in China: pathogenesis and correlates of fatal outcome. Clin Infect Dis. 2012;54(4):527-533.

12. Deng B, et al. Clinical features and factors associated with severity and fatality among patients with severe fever with thrombocytopenia syndrome Bunyavirus infection in Northeast China. PLOS ONE. 2013;8(11):e80802.

13. Shin J, Kwon D, Youn SK, Park JH. Characteristics and factors associated with death among patients hospitalized for severe fever with thrombocytopenia syndrome, South Korea, 2013. Emerging Infect Dis. 2015;21(10):1704-1710.

14. Matsuno K, et al. Animal models of emerging tick-borne phleboviruses: determining target cells in a lethal model of SFTSV infection. Front Microbiol. 2017;8:104.

15. Li H, et al. Epidemiological and clinical features of laboratory-diagnosed severe fever with thrombocytopenia syndrome in China, 2011-17: a prospective observational study. Lancet Infect Dis. 2018;18(10):1127-1137.

16. Sun Y, et al. Host cytokine storm is associated with disease severity of severe fever with thrombocytopenia syndrome. J Infect Dis. 2012;206(7):1085-1094.

17. Yoo JR, Kim SH, Kim YR, Lee KH, Oh WS, Heo ST. Application of therapeutic plasma exchange in patients having severe fever with thrombocytopenia syndrome. Korean J Intern Med. 2019;34(4):902-909.

18. Kaneko M, et al. A patient with severe fever with thrombocytopenia syndrome and hemophagocytic lymphohistiocytosis-associated involvement of the central nervous system. J Infect Chemother. 2018;24(4):292-297.

19. Kwon JS, et al. Kinetics of viral load and cytokines in severe fever with thrombocytopenia syndrome. JClin Virol. 2018;101:57-62.

20. Qu B, et al. Suppression of the interferon and $\mathrm{NF}-\kappa \mathrm{B}$ responses by severe fever with thrombocytopenia syndrome virus. J Virol. 2012;86(16):8388-8401.

21. Sun Q, et al. Host responses and regulation by $\mathrm{NF} \kappa \mathrm{B}$ signaling in the liver and liver epithelial cells infected with a novel tick-borne Bunyavirus. Sci Rep. 2015;5:11816.

22. Tani H, et al. Efficacy of T-705 (favipiravir) in the treatment of infections with lethal severe fever with thrombocytopenia syndrome virus. mSphere. 2016;1(1):e00061-15.

23. Moriyama M, Igarashi M, Koshiba T, Irie T, Takada A, Ichinohe T. Two conserved amino acids within the NSs of severe fever with thrombocytopenia syndrome phlebovirus are essential for anti-interferon activity. J Virol. 2018;92(19):e00706-18.

24. Tani H, et al. Therapeutic effects of favipiravir against severe fever with thrombocytopenia syndrome virus infection in a lethal mouse model: Dose-efficacy studies upon oral administration. PLoS ONE. 2018;13(10):e0206416.

25. Yamada S, et al. RIG-I-like receptor and Toll-like receptor signaling pathways cause aberrant production of inflammatory cytokines/chemokines in a severe fever with thrombocytopenia syndrome virus infection mouse model. J Virol. 2018;92(13):e02246-17.

26. Li XK, et al. Arginine deficiency is involved in thrombocytopenia and immunosuppression in severe fever with thrombocytopenia syndrome. Sci Transl Med. 2018;10(459):eaat4162.

27. Song P, et al. Deficient humoral responses and disrupted B-cell immunity are associated with fatal SFTSV infection. Nat Commun. 2018;9(1):3328.

28. Paessler S, Walker DH. Pathogenesis of the viral hemorrhagic fevers. Annu Rev Pathol. 2013;8:411-440.

29. Liu Y, Wu B, Paessler S, Walker DH, Tesh RB, Yu XJ. The pathogenesis of severe fever with thrombocytopenia syndrome virus infection in alpha/ beta interferon knockout mice: insights into the pathologic mechanisms of a new viral hemorrhagic fever. J Virol. 2014;88(3):1781-1786.

30. Jin C, et al. Pathogenesis of emerging severe fever with thrombocytopenia syndrome virus in C57/BL6 mouse model. Proc Natl Acad Sci USA. 2012;109(25):10053-10058.

31. Park SJ, et al. Ferret animal model of severe fever with thrombocytopenia syndrome phlebovirus for human lethal infection and pathogenesis. Nat Microbiol. 2019;4(3):438-446.

32. Martines RB, Ng DL, Greer PW, Rollin PE, Zaki SR. Tissue and cellular tropism, pathology and pathogenesis of Ebola and Marburg viruses. JPathol. 2015;235(2):153-174.

33. Zaki S, et al. CDC Grand Rounds: discovering new diseases via enhanced partnership between public health and pathology experts. MMWR Morb Mortal Wkly Rep. 2014;63(6):121-126.

34. Hiraki T, et al. Two autopsy cases of severe fever with thrombocytopenia syndrome (SFTS) in Japan: a pathognomonic histological feature 
and unique complication of SFTS. Pathol Int. 2014;64(11):569-575.

35. Kim N, et al. Bone marrow findings in severe fever with thrombocytopenia syndrome: prominent haemophagocytosis and its implication in haemophagocytic lymphohistiocytosis. J Clin Pathol. 2016;69(6):537-541.

36. Uehara N, Yano T, Ishihara A, Saijou M, Suzuki T. Fatal severe fever with thrombocytopenia syndrome: an autopsy case report. Intern Med. 2016;55(7):831-838.

37. Li S, et al. Multiple organ involvement in severe fever with thrombocytopenia syndrome: an immunohistochemical finding in a fatal case. Virol J. 2018;15(1):97.

38. Cattoretti G, Shaknovich R, Smith PM, Jäck HM, Murty VV, Alobeid B. Stages of germinal center transit are defined by B cell transcription factor coexpression and relative abundance. J Immunol. 2006;177(10):6930-6939.

39. Tani H, et al. Characterization of glycoproteinmediated entry of severe fever with thrombocytopenia syndrome virus. J Virol. 2016;90(11):5292-5301.

40. Shaffer AL, Rosenwald A, Staudt LM. Lymphoid malignancies: the dark side of B-cell differentiation. Nat Rev Immunol. 2002;2(12):920-932.

41. Mine S, et al. Interleukin-6-dependent growth in a newly established plasmablastic lymphoma cell line and its therapeutic targets. Sci Rep. 2017;7(1):10188.

42. Stokes RW, Doxsee D. The receptor-mediated uptake, survival, replication, and drug sensitivity of Mycobacterium tuberculosis within the mac- rophage-like cell line THP-1: a comparison with human monocyte-derived macrophages. Cell Immunol. 1999;197(1):1-9.

43. Fink K. Origin and function of circulating plasmablasts during acute viral infections. Front Immunol. 2012;3:78.

44. Lanzavecchia A. Dissecting human antibody responses: useful, basic and surprising findings. EMBO Mol Med. 2018;10(3):e8879.

45. Garcia-Bates TM, et al. Association between magnitude of the virus-specific plasmablast response and disease severity in dengue patients. J Immunol. 2013;190(1):80-87.

46. McElroy AK, et al. Human Ebola virus infection results in substantial immune activation. Proc Natl Acad Sci USA. 2015;112(15):4719-4724.

47. Kaminski DA, Wei C, Qian Y, Rosenberg AF, Sanz I. Advances in human B cell phenotypic profiling. Front Immunol. 2012;3:302.

48. Kwissa M, et al. Dengue virus infection induces expansion of a CD14(+)CD16(+) monocyte population that stimulates plasmablast differentiation. Cell Host Microbe. 2014;16(1):115-127.

49. Appanna R, et al. Plasmablasts during acute dengue infection represent a small subset of a broader virus-specific memory B cell pool. EBioMedicine. 2016;12:178-188.

50. Kuka M, Iannacone M. Viral subversion of B cell responses within secondary lymphoid organs. Nat Rev Immunol. 2018;18(4):255-265.

51. Zhang J, Yan X, Li Y, Gao R, Wang P, Mo W. Reactive plasmacytosis mimicking multiple myeloma associated with SFTS virus infection: a report of two cases and literature review. BMC Infect Dis.
2018;18(1):528

52. Takahashi T, et al. Transient appearance of plasmablasts in the peripheral blood of Japanese patients with severe fever with thrombocytopenia syndrome. J Infect Dis. 2019;220(1):23-27.

53. Ponzoni M, et al. Definition, diagnosis, and management of intravascular large B-cell lymphoma: proposals and perspectives from an international consensus meeting. JClin Oncol. 2007;25(21):3168-3173.

54. Fukuma A, et al. Severe fever with thrombocytopenia syndrome virus antigen detection using monoclonal antibodies to the nucleocapsid protein. PLoS Negl Trop Dis. 2016;10(4):e0004595.

55. Takahira H, et al. Establishment of a human myeloma cell line with growth-promoting activity for bone marrow-derived fibroblastoid colony-forming cells. Exp Hematol. 1994;22(3):261-266.

56. Katano $\mathrm{H}$, et al. Establishing and characterizing a CD30-positive cell line harboring HHV-8 from a primary effusion lymphoma. J Med Virol. 1999;58(4):394-401.

57. Renne R, et al. Lytic growth of Kaposi's sarcomaassociated herpesvirus (human herpesvirus 8) in culture. Nat Med. 1996;2(3):342-346.

58. Taniguchi S, Fukuma A, Tani H, Fukushi S, Saijo M, Shimojima M. A neutralization assay with a severe fever with thrombocytopenia syndrome virus strain that makes plaques in inoculated cells. J Virol Methods. 2017;244:4-10.

59. Yanagisawa Y, et al. Effusion and solid lymphomas have distinctive gene and protein expression profiles in an animal model of primary effusion lymphoma. J Pathol. 2006;209(4):464-473. 